\title{
Dynamically adaptive data-driven simulation of extreme hydrological flows
}

\author{
Pushkar Kumar Jain ${ }^{\mathrm{a}, *}$, Kyle Mandli ${ }^{\mathrm{b}}$, Ibrahim Hoteit ${ }^{\mathrm{c}}$, Omar Knio ${ }^{\mathrm{d}}$, Clint Dawson ${ }^{\mathrm{e}}$ \\ ${ }^{a}$ Department of Aerospace Engineering and Engineering Mechanics, University of Texas at Austin, 210 E \\ 24th St, Austin, TX 78712-1085, USA \\ ${ }^{b}$ Department of Applied Physics and Applied Mathematics, Columbia University, 500 W. 120th St., New \\ York, NY 10027, USA \\ ${ }^{c}$ Division of Physical Sciences and Engineering, King Abdullah University of Science and Technology, \\ Thuwal, Saudi Arabia \\ ${ }^{d}$ Division of Computer, Electrical and Mathematical Sciences and Engineering, King Abdullah University \\ of Science and Technology, Thuwal, Saudi Arabia \\ ${ }^{e}$ Institute for Computational Engineering and Science, University of Texas at Austin, 201 E 24th St. Stop \\ C0200, Austin, TX 78712-1229, USA
}

\begin{abstract}
Hydrological hazards such as storm surges, tsunamis, and rainfall-induced flooding are physically complex events that are costly in loss of human life and economic productivity. Many such disasters could be mitigated through improved emergency evacuation in real-time and through the development of resilient infrastructure based on knowledge of how systems respond to extreme events. Data-driven computational modeling is a critical technology underpinning these efforts. This investigation focuses on the novel combination of methodologies in forward simulation and data assimilation. The forward geophysical model utilizes adaptive mesh refinement (AMR), a process by which a computational mesh can adapt in time and space based on the current state of a simulation. The forward solution is combined with ensemble based data assimilation methods, whereby observations from an event are assimilated into the forward simulation to improve the veracity of the solution, or used to invert for uncertain physical parameters. The novelty in our approach is the tight two-way coupling of AMR and ensemble filtering techniques. The technology is tested using actual data from the Chile
\end{abstract}

\footnotetext{
* Corresponding author

Email addresses: pushkarjain1991@utexas.edu (Pushkar Kumar Jain),

kyle.mandli@columbia.edu (Kyle Mandli), ibrahim.hoteit@kaust. edu.sa (Ibrahim

Hoteit), omar.knio@kaust. edu.sa (Omar Knio), clint@ices.utexas.edu (Clint Dawson)
} 
tsunami event of February 27, 2010. These advances offer the promise of significantly transforming data-driven, real-time modeling of hydrological hazards, with potentially broader applications in other science domains.

Keywords: Data assimilation, Ensemble Kalman filter, Adaptive mesh refinement, Tsunami, Okada model, Shallow water equations, Uncertainty quantification

\section{Introduction}

Coastal areas are often densely populated [1] and are most vulnerable to extreme hazards such as storm surges, hurricanes and tsunamis. As of the 2010 census, $39 \%$ of the US population or 123.3 million people are concentrated in areas directly on the shoreline [2]. Furthermore, the world coastal population is projected to rise to around 949 million (then projected global population is 8.7 billion) by 2030, and to 1.4 billion people (then projected global population is 11.3 billion) by 2060 [3]. This means that a large portion of the global population is vulnerable to the destruction that may be caused by these extreme events. The effects of hazardous flows can be mitigated by using reliable computational fluid dynamics models that can accurately forecast the events and disseminate information quickly, to aid in preparedness and timely preemptive measures when necessary.

In this paper, we investigate computational algorithms to advance the science of real-time prediction of coastal and overland hydrological hazards. The numerical models that simulate large scale geophysical flows involve solving simultaneous partial differential equations that govern the flow of fluids. Under certain assumptions, the governing equations can be used to simulate flows on a synoptic scale, replicating the path and movement of extreme hydrological flows such as hurricanes and tsunamis [47]. The input to these models are in the form of initial condition of the flow, boundary conditions, forcing parameters such as wind stresses, topography of sea bed, salinity etc. Simulations of hydrological hazards are computationally challenging in at least two aspects: they are inherently multi-scale (both spatially and temporally) [8, 9], and there are significant uncertainties in the underlying physical models [10-12]. These uncertainties are inevitable. This means that uncertainty in any of the inputs can lead 
to solutions that may not replicate reality perfectly. For instance, in tsunami modeling, the sea floor motion that generates the tsunami is not well determined because the fault displacement is dependent on the subsurface geologic structure. A good measure is to assess the results through validation with field data. This can be in the form of satellite imagery or data from buoys in oceans. However, measurements are not only scarce, but also carry some uncertainties due to various factors such as sensor precision or observation location.

Data assimilation is a technique whereby the observations along with the uncertainties are combined with the forward models in a statistical way to compute the best possible estimate of the state system. There are two main approaches to assimilating data into numerical models - 1) variational methods such as 3D-Var [13] and 4D-Var [14] and 2) sequential methods [15, 16]. This paper uses the two step sequential approach based on the Kalman filter as proposed by [15, 16] in which data are incorporated as they are available. The first step is forecasting using the forward model, followed by an analysis step, statistically averaging the observation with the model forecast according to their estimated uncertainties. For linear systems, under the assumption of unbiased model and incoming uncorrelated Gaussian distributed observation and model errors, the method yields the best linear unbiased estimate. For non-linear dynamics, the Extended Kalman filter was proposed in [17]. These methods, namely Kalman and Extended Kalman filters, propagate the state of the system as well as the associated error covariance, a measure of the uncertainty in the state estimate. Propagation of the error covariance can be computationally prohibitive when the dimension of the state is large. This is typical for large scale hydrodynamic flows, where the dimension of the state can be $\mathcal{O}\left(10^{8}\right)$ [18]. This issue is addressed by ensemble based methods such as the Ensemble Kalman filter [19, 20]. These schemes use Monte Carlo methods to estimate the background error covariance. The method performs an ensemble run of the forward model in the forecast stage from which the sample covariance is calculated and then used at the analysis step. The ensemble based technique asymptotically approaches the Kalman filter in the limit of large ensembles and Gaussian error distribution [21]. The computational cost has been well described in [20].

Over the years, a variety of ensemble filters have been developed and implemented 
in various fields [22,-27]. Several review articles [20, 28,-32] enumerate the advancements in the field, leading to development of various filters, such as optimal interpolation [33], EnSRF [34], EAKF [35, 36], SEIK [37, 38], ETKF [39], SEEK [40, 41], RRSQRT [42], ESTKF [43]. Many of these filters have been developed with the underlying idea of a low-rank approximation of the error covariance matrix.

One of the challenges of data assimilation is that factors such as neglected uncertainties, poorly known model errors and a low rank representation of the estimation error via a finite ensemble size lead to underestimation of the error covariances of the filter. The underestimation of the variance is mitigated by the use of covariance inflation [44-47]. In addition, localization can be applied to address rank deficiency and spuriously large cross-covariances between different state variables. Two methods of localization often used are 1) Covariance localization [48], where the error covariance matrix is transformed based on physical distance of grid points in the numerical model, and 2) Domain localization [49], where the physical domain is divided into individual local domains for updates. Also, [50] demonstrates a regulated localization scheme that weighs elements of observation error covariance matrix by a localization function of varying width.

This paper aims to develop a data assimilation framework that employs the above described aspects of ensemble based filters with large scale hydrodynamic models, and effectively improve forecasting. Ensemble based filters have been implemented in various large scale oceanic models [26, 51,-54]. [31] reviews the developments in regional data assimilation over the last 15 years. With respect to employing data assimilation with tsunami simulations, significant literature exists. [55] discusses the need and challenges of real time tsunami forecasting with respect to accuracy, speed and robustness of tsunami warning systems. [56] assumes a linear system and assimilates data using optimal interpolation for the 2012 Haida Gwaii earthquake. In this study, the Kalman gain (called the smoothing matrix) does not change with time and is a function of the station distribution. [57, 58] employed the particle filter method to assimilate tide gauge data into a tsunami simulation model. As part of the NOAA tsunami forecasting system [59], the best estimates of tsunami amplitudes and arrival times for potential at-risk communities are obtained by assimilating real-time event data with the Method 
of Splitting Tsunami (MOST) model [60, 61].

Some of these large scale ocean models employ adaptive mesh refinement (AMR) technique. Adaptive meshing uses algorithms that dynamically refine the grids spatially, temporally or both, to obtain fine scale solutions in the areas of interest that can be identified based on user criteria [62,65]. There are two main approaches of implementing AMR. Both the methods identify the regions where fine scale solutions are of interest. One class of AMR technique splits the elements of the grid into finer elements on the same grid [66]. Another class of AMR techniques involve multi-level mesh structure that is comprised of various refinement levels [64, 67]. AMR technique proves to be advantageous towards reducing computational cost. In large scale hydrodynamic models, AMR can be used to dynamically obtain fine scale solutions near the coast, where we are most interested in the solution, while retaining a coarse mesh in deep oceans. For instance, in the case of tsunami simulations, we can dynamically refine the mesh along the moving wave front [68, 69].

In the area of data assimilation with AMR, several methods involving 3D-Var and 4D-Var have been proposed by [70, 71]. In a very recent study, [72] implemented the ensemble based filter for data assimilation. The ensembles were interpolated to a reference mesh, while accounting for the interpolation errors using conservative interpolation operators. However, no prior work has analyzed the implementation of ensemble based filter for data assimilation with multi-level AMR, and this paper is the first attempt to provide primary insights in this direction. Our goal is to build an advanced data-driven AMR model for efficient simulation and forecasting of coastal marine dynamics.

In this paper, we describe the traditional ensemble Kalman filter and an AMR capable numerical model - GEOCLAW. Coupling ensemble based assimilation with AMR can be challenging as the AMR algorithm may dictate the ensemble members to adapt in their own way. This makes it difficult to compute quantities such as the mean and the error covariance of the state vector, essential for the assimilation step. We discuss the hurdles faced in detail and devise a methodology for a suitable solution. In the process, we define the assimilation field and the observation operator. We propose a methodology wherein data are assimilated at all the multi-scale levels. Further, we ap- 
ply the data assimilation framework on tsunami models and demonstrate an algorithm for the initial ensemble generation, which is essential for any ensemble based filtering technique. The final sections put forth validation results from a real event - the Chile tsunami of February 27, 2010. We perform twin experiments by testing the assimilation process with synthetic measurements. The experiments will look into the aspects of localization radius, observation configurations, minimum ensemble size and the initial ensemble affecting state estimates. This is followed by an AMR-coupled assimilation run of the observed real event, taking into account the available gauge data.

\section{Ensemble based Kalman filters}

The traditional ensemble Kalman filter EnKF described in [19, 20] is formulated as a sequential process of the forecast step followed by the analysis step. Unlike EnKF, filters such as SEIK [38] and ETKF [39] are implemented as ensemble square-rootfilters that split the analysis step into a state update step and a resampling step. The state update step computes the analysis state and its error covariance matrix and the resampling step generates the ensemble members that exactly represent the analysis state and its error covariance matrix. In the current study, we have implemented the Error Subspace Transform Kalman filter (ESTKF) [43], a variant of the SEIK filter that performs ensemble transformation in the error subspace. The ESTKF combines the advantages of SEIK filter and ETKF [43]. ESTKF computes ensemble transformations that are identical to ETKF, but at a lower computational cost. Further, unlike the SEIK filter, the ensemble transformation in ESTKF is independent of the order of ensemble members in the ensemble matrix. This was demonstrated in the studies 1 where the applications are close enough to the current one. This section outlines the ESTKF algorithm that is described in detail in ([43]).

Consider a dynamical system $M_{k}$ that forecasts the true state from time $t_{k}$ to $t_{k+1}$ as,

$$
x_{k}^{t}=M_{k}\left(x_{k-1}^{t}\right)+\eta_{k},
$$

\footnotetext{
/http://pdaf.awi.de/trac/wiki/WhichFiltertouse
} 
where $x_{k}^{t}$ is the true state and $\eta_{k}$ is the system noise at time $t_{k}$ assumed to be unbiased. Further, consider the observation $y_{k}$ of the state at time $t_{k}$ given by,

$$
y_{k}=H_{k} x_{k}^{t}+\epsilon_{k}
$$

where $\epsilon_{k}$ is the measurement error at time $t_{k}$ assumed to be unbiased. $H_{k}$ is the operator that maps the field of interest from the state space to the observation space. With an ensemble of size $N$, we define the forecasted state matrix $X_{k}^{f}$ as,

$$
X_{k}^{f}=\left[\begin{array}{ccccc}
\vdots & & \vdots & & \vdots \\
x_{k, 1}^{f} & \ldots & x_{k, p}^{f} & \ldots & x_{k, N}^{f} \\
\vdots & & \vdots & & \vdots
\end{array}\right],
$$

where $x_{k, p}^{f}$ is the $p^{t h}$ forecasted ensemble member at time $t_{k}$. We also define $\bar{X}_{k}^{f}=$ $\bar{x}_{k}^{f} \mathbf{1}_{\mathbf{d}}$, where the mean of the forecasted ensemble states is $\bar{x}_{k}^{f}=\frac{1}{N} \sum_{i=1}^{i=N} x_{k, i}^{f}$ and $\mathbf{1}_{\mathbf{d}}$ is the matrix such that all the entries are $1 s$. At time $t_{k}, P_{k}^{f}$ is the forecast error covariance and $R_{k}=\epsilon_{k} \epsilon_{k}^{T}$ is the observation error covariance. The observation error comprises of two components, the measurement error and the representation error [73]. For the various test cases discussed later in the paper, we assume that both the components have been accounted by a constant root mean square error value. Similar to the forecast variables defined above, we define $X_{k}^{a}$ as the analysis state matrix at time $t_{k}$ comprising of the analysis state ensemble members $x_{k, p}^{a}$. In ESTKF, the forecast error covariance matrix at time $t_{k}$ is represented as,

$$
P_{k}^{f}=L_{k} G L_{k}^{T},
$$

where,

$$
\begin{aligned}
& L_{k}=X_{k}^{f} \Omega, \\
& G=\frac{1}{N-1} \mathbb{I}_{(N-1) \times(N-1)}, \\
& \Omega_{i, j}= \begin{cases}1-\frac{1}{N} \frac{1}{\frac{1}{\sqrt{N}}+1} & , i=j, i<N \\
-\frac{1}{N} \frac{1}{\sqrt{\sqrt{N}}+1} & , i \neq j, i<N . \\
-\frac{1}{\sqrt{N}} & , i=N\end{cases}
\end{aligned}
$$


$\Omega_{i, j}$ is the projection matrix that has a full rank, zero column sums with the columns being orthonormal. I is the Identity matrix. The state vector $X_{k}^{f} \in \mathbb{R}^{n \times N}, P_{k}^{f} \in \mathbb{R}^{n \times n}$, $L_{k} \in \mathbb{R}^{n \times(N-1)}, G \in \mathbb{R}^{(N-1) \times(N-1)}$ and $\Omega \in \mathbb{R}^{N \times(N-1)}$. The ESTKF algorithm can be outlined as follows:

1. Forecasting from $t_{k-1}$ to $t_{k}$ :

Starting from a given analysis state matrix $X_{k-1}^{a}$, integrate each ensemble member $x_{k-1, p}^{a}$, with the dynamical system $M_{k}$ as,

$$
x_{k, p}^{f}=M_{k}\left(x_{k-1, p}^{a}\right) .
$$

2. Updating at time $t_{k}$ :

Compute the analysis state estimate $\bar{x}_{k}^{a}$ as,

$$
\bar{x}_{k}^{a}=\bar{x}_{k}^{f}+L_{k} A_{k}\left(H_{k} L_{k}\right)^{T} R_{k}^{-1}\left(y_{k}-H_{k} \bar{x}_{k}^{f}\right),
$$

where $A_{k}$ is the transform matrix given by,

$$
A_{k}^{-1}=\rho(N-1) \mathbb{I}+\left(H_{k} L_{k}\right)^{T} R_{k}^{-1}\left(H_{k} L_{k}\right) .
$$

$\rho$ is the inflation factor used to enhance the filter robustness and to partially account for model errors [38, 40]. More sophisticated methods to treat model errors have been discussed in [74].

3. Resampling:

This step is the second order exact sampling that computes the ensemble states.

$$
X_{k}^{a}=\bar{x}_{k}^{a} \mathbf{1}_{d}+\sqrt{N-1} X_{k}^{f} \Omega C_{k} \Omega^{T} .
$$

where $C_{k}$ is the square root of $A_{k}$.

\section{Model description: GEOCLAW}

The mathematical model used is the 2D depth averaged shallow water equations in $x$ and $y$ directions, governed by,

$$
h_{t}+(h u)_{x}+(h v)_{y}=0,
$$




$$
\begin{aligned}
& (h u)_{t}+\left(h u^{2}+1 / 2 g h^{2}\right)_{x}+(h u v)_{y}=-g h B_{x}-D u, \\
& (h v)_{t}+(h u v)_{x}+\left(h v^{2}+1 / 2 g h^{2}\right)_{y}=-g h B_{y}-D v,
\end{aligned}
$$

where $h$ is the total depth of water column, $u$ and $v$ are depth averaged velocities in the $x$ and $y$ directions respectively, $B$ is the bathymetry, $D$ is the drag coefficient and $g$ is the acceleration due to gravity.

The equations are solved using GEOCLAW, an open-source package written in Fortran [69], with a user interface in Python. GEOCLAW is a part of the general Clawpack (Conservation Laws) package [68]. It uses high-resolution shock-capturing finite volume methods on logically Cartesian grids to solve non-linear hyperbolic systems of partial differential equations [75].

GEOCLAW uses AMR algorithms as discussed in [69, 75-77], to simulate multiscale geophysical flows. AMR is implemented using a patch-based mesh refinement approach, shown in Figure 1. where multiple levels of structured meshes are used to reach a fine resolution [64]. For instance, mesh levels can be created that can range from a few meters at the coastline, to a few hundred kilometers in the deep ocean.

For notation, a Level $k$ refinement is called a $k^{\text {th }}$ level patch. Thus, the coarsest mesh is called a Level 1 patch. For tsunami modeling, GeOCLAW has the capability to use anisotropic refinement in time as well.

\section{Coupling GeoClaw and data assimilation}

This section describes the challenges faced in the development of the online coupled system of GEOCLAW with the ensemble assimilation techniques and presents proposed solutions. Section 4.1 addresses the "supermesh" methodology used for handling the ensemble members with different AMR structure, which is essential for the assimilation step. Section 4.2 describes the quantity of interest for assimilation and the construction of the state vector. Section 4.3 describes the construction of the observation operator.

\subsection{Mesh union strategy}

The primary difficulty in using AMR in conjunction with ensemble filtering schemes is that the regridding operation during the forecast allows every ensemble member to 
adapt its grid in its own way; hence, at the end of the forecast stage, one ensemble member may obtain a grid structure that is different from the other ensemble members. Figure 1 illustrates a hypothetical scenario of two ensemble members at an analysis stage, with different refined patches at level 2 and level 3. Thus, at finer levels, the ensemble members have varying number of cells and thereby end up with a varying state vector length. This means that statistical quantities such as covariance of the state, essential for the update step, are no longer straightforward to compute.

This motivates us to develop a methodology that can enforce all the ensemble members to produce the same mesh structure at all levels at the analysis stage. At the assimilation stage, (1) construct a union of meshes of all the ensemble members, also called a supermesh, (2) communicate the information of every ensemble member to the supermesh, (3) perform analysis, (4) communicate the updated information back from the supermesh to the respective ensemble member, and (5) continue the forecast.

Remark i - Construction of a supermesh is done by modifying the default regridding operation to a constrained regridding operation. The mesh structure of each ensemble member is communicated to every other ensemble member. Thus, at any given point in the domain, we know the maximum refinement level that exists for at least one ensemble member. Formally, let $\phi_{(x, y)}^{(i)}$ and $\delta_{(x, y)}^{(i)}$ be the maximum refinement level at a point $(x, y)$ for the $i^{t h}$ ensemble member, before and after the analysis step respectively. That is,

$$
\delta_{(x, y)}^{(i)} \triangleq \max _{i}\left(\phi_{(x, y)}^{(i)}\right)
$$

After the analysis step, the mesh structure for all the ensemble members is the same. Hence, $\delta_{(x, y)}^{(i)} \equiv \delta_{(x, y)}$. This way we ensure that during the assimilation, all the members have the same mesh structure at the start of the assimilation step.

Remark ii - AMR algorithm in GEOCLAW constructs a finer mesh to Level $k+1$ by first flagging cells in Level $k$. At Level $k$, the flagged cells are clustered based on a clustering parameter that defines the cut-off percentage of flags required to bound the flags within a rectangular region and construct a patch [78]. This implies that the union of two ensemble members may not lead to an "exact union“. Nevertheless, since all the ensemble members have the same flags at all levels, the deterministic nature of the 
clustering algorithm guarantees the same finer patches for the finer levels. When this procedure is applied recursively from coarsest mesh to finer mesh, all the ensemble members end up with the same mesh structure.

Remark iii - A regrid operation in the forecast step (after assimilation) removes the unnecessary refinement and the ensemble members undergo forecasting in the usual manner thereafter.

Remark iv - In practice, it is observed that the mesh structures of the ensemble members do not vary in high degree from each other.

Implementation of the above strategy of the union of meshes leads to the final mesh shown in Figure 1, on which assimilation will be performed.

Here we illustrate the generation of the supermesh grid via a GEOCLAW run of a model problem that simulates the water flow in a parabolic bowl. The initial condition of the water surface elevation is a Gaussian hump shown in Figure 2. We run the simulation for 3.2 seconds. The maximum AMR level that is possible during the simulation is set to 2 . We generate two ensemble members with different initial amplitudes. Figure 3 shows the snapshots of water surface elevation at instances when the supermesh is formed. The left and the middle column represent the two ensemble members, and the right column is the case if the supermesh was formed. In the figure, the rectangular patches without any cells (clean patches) are the level 2 patches. It is seen that from 2.56 seconds, as the water front is moving radially outward, different initial amplitudes causes the ensemble members to adapt with different meshes, forming a supermesh accordingly. As mentioned in Remark ii above, the supermesh grid structure is not a perfect union of grids of the ensemble members.

\subsection{Construction of state vector}

For the current study, the state vector is constructed in a way that all the cells at all the levels of the supermesh are combined into one single array. We define a patch as the collection of grids at a given level. If $P$ is the maximum refinement level in $\delta$, then 
we define the state to be assimilated $(\psi)$, water surface elevation as

$$
\psi \triangleq\left[\begin{array}{c}
\text { level } 1 \text { patch } \\
\vdots \\
\text { level } k \text { patch } \\
\vdots \\
\text { level P patch, }
\end{array}\right]
$$

where we have augmented the state vector at various levels into a new state vector. For instance, the state vector constructed from the hypothetical union of mesh in Figure 1 would be of size $64+112+160=336$. This means that if our state vector has a cell value at a finer level, then it also contains the underlying coarser cell value.

\subsection{Construction of observation operator}

In a multi-level AMR structure, a measurement may come from a location where more than one level of refinement exists. A measurement recorded at a physical location that belongs to the finest cell will also belong to the underlying coarse cells. Given that the state vector combines the variables from all the levels at a given physical location, the observation operator is constructed in such a way that the same observation realization is used in all the levels at that physical location.

For instance, consider the mesh structure in Figure 4 that represents a single coarse cell denoted by $v_{1}$ at Level 1 . The coarse cell $v_{1}$ is refined to finer cells denoted by $v_{2}, v_{3}, v_{4}, v_{5}$ at Level 2. The cell $v_{2}$ is further refined to cells $v_{6}, v_{7}, v_{8}, v_{9}$ at Level 3. The state vector for this simple structure is $\psi=\left[v_{1}, v_{2}, \ldots, v_{8}, v_{9}\right]^{T}$. Depending on the location of the incoming measurement, multiple scenarios occur. Scenario 1 - If there exists just a single incoming observation $M_{1}$ at a point belonging in cell $v_{6}$, then clearly, $M_{1}$ belongs to $v_{2}$ and $v_{1}$ as well. Hence the measurement recorded for $v_{1}, v_{2}$ and $v_{6}$ is $M_{1}$. Scenario 2 - If there exists two incoming observations $M_{1}$ and $M_{2}$ at the locations belonging to cells $v_{6}$ and $v_{9}$, respectively, then both the measurement values belong to $v_{2}$ and $v_{1}$ as well. Hence, the measurement values recorded at $v_{6}$ is $M_{1}$, at $v_{9}$ is $M_{2}$ and at $v_{1}$ and $v_{2}$ is taken as the average of $M_{1}$ and $M_{2}$. This idea can be extended to other scenarios as well. 


\subsection{Data assimilation framework}

We have used the data assimilation software - Parallel Data Assimilation Framework (PDAF) [79], a Fortran library for various ensemble filters such as EnKF, ETKF, ESTKF, SEIK, SEEK and the localized filters such as LETKF, LSEIK, LESTKF. The library supports parallel ensemble forecasts and assimilation in MPI and OpenMP. As seen in Figure 5, each GEOCLAW run is a serial run representing a single ensemble member. The forecast of the ensemble members are executed in parallel under MPI parallelism. For the assimilation stage, the forecasted state vectors are gathered to the root for the update. The updated states are scattered back to the cluster for next forecast stage. For the current study, a single ensemble member is assigned to a single core during the forecasting. Hence, a total of $N$ ensemble members is performed by $N$ cores in a cluster, wherein, each core is assigned to one ensemble member. The assimilation is performed by a single core as well. It should be noted that the PDAF library has the capability to adjust the number of cores per ensemble member during the forecast stage and the number of cores in the update step. The simulations were carried on a local desktop with 8 cores and not in a distributed environment. A virtual MPI environment was created such that the 8 cores would mimic the user-defined number of cores. Hence, the wall clock time will not be a good estimate for analyzing execution time. In the present study, the coupling is done in such a way that the forecasts are distributed amongst the processors and the analysis is done by a single processor.

\section{Initial ensemble generation}

\subsection{Motivation}

Generating a representative initial ensemble to start the assimilation systems is important to obtain good performance with EnKF. Several approaches have been proposed [37, 80, 81]. For instance, [37] discusses the idea of generating the initial ensemble for large scale ocean and atmosphere models via second-order exact sampling from a model trajectory that is obtained from years of spin-up. However, unlike the ocean and atmospheric models, where the initial ensemble of states can be produced via model spin-ups, tsunamis occur relatively instantly due to the rapid bathymetry changes. We 
address this inability to generate ensembles via model spin-ups by an algorithm that can effectively map the uncertainty in the bathymetry changes at the event of tsunami to the initial ensemble.

The tsunami simulation models use bathymetry changes due to the associated earthquake. For instance, the Okada model [82] relates the topography change $\Delta d$, to various fault parameters,

$$
\Delta d \sim f(\text { strike, length, width, depth, slip, rake, dip, longitude, latitude). }
$$

This means that any uncertainty in the fault parameters can produce the possible bathymetry changes that will in turn produce the subspace of initial states of the tsunami.

\subsection{Procedure}

We assume that the fault parameters have a multivariate Gaussian distribution with a specified mean and a covariance. We also assume that the fault parameters are not correlated to each other and hence the covariance matrix is diagonal with each entry representing the variance of a fault parameter. Under these assumptions, first perturb the fault parameters using a relatively large sample $N_{s}$. The subspace of the perturbations generated, represents the possible bathymetry deformations. Thus, a forward integration of the model up to a small time (called checkpoint time) with each of the samples as the initial condition, generates the subspace of initial tsunamis. Similar to the idea proposed by [37, 81], we adopt second-order exact sampling from various model states. But unlike the temporal snapshots of the states, we use the snapshots of the various possible tsunamis that we have generated, to develop a perturbation matrix of a desired size. These perturbations can then be later added to the mean water surface elevation at the checkpoint time and in turn generate the initial ensemble.

\subsection{Practical implementation}

Large scale ocean model softwares use checkpoint and restart capabilities, wherein, a forecast can be resumed from a checkpoint time. We rely on this capability to implement the initial ensemble generation algorithm. Let us denote $t_{o}, t_{c p}$ and $t_{e n d}$ as the initial time, the checkpoint time and the simulation final time respectively. 
1. Perturbation generation (Checkpoint run) - At time $t_{o}=0$, draw a relatively large random sample (say $N_{s}=100$ ) of the fault parameters of the Okada model, under the assumption that the parameters are uncorrelated to each other and are normally distributed with a user specified mean and a diagonal covariance. Integrate using each of the fault up to a reasonably small time, called the checkpoint time $t_{c p}$ to generate the subspace of possible tsunamis.

2. Use the 100 model states $\left(N_{s}\right)$ and a user desired ensemble size $\left(n_{s}\right)$ to generate the perturbation matrix. Add the perturbations to the mean water surface elevation at time $t_{c p}$.

3. Assimilation run - With the assimilation facility activated, resume the code at the checkpoint time $t_{c p}$ till the final time $t_{e n d}$.

Remark i - For a given problem, the eigen decomposition has to be performed only once at the initial time $t_{o}=0$. The eigenvectors and the values are small in size and can be stored on disk. This can be a part of the preprocessing stage.

Remark ii - A small checkpoint time $t_{c p}$ implies that forecasting $N_{s}$ (here 100) ensemble members till the checkpoint time is not so computationally expensive.

\section{Assimilation experiments on Chile 2010 tsunami event}

On February 27, 2010 at 03:34 local time (06:34 UTC), an 8.8 magnitude earthquake off the coast of Chile triggered a massive tsunami, causing catastrophic damage to life and property.

In this section, we will apply the discussed methodologies of initial ensemble generation and AMR coupled data assimilation framework on a test setup that simulates the event. We first describe the model parameters for simulating the actual tsunami event. To assess the assimilation system, we perform twin experiments, wherein we treat the above run as the "truth" and use the water surface elevation data as measurement values for the assimilation runs. We will demonstrate that assimilation is effective on model runs that have the initial conditions that are different from the true initial conditions. Note that the initial condition here refers to the fault parameters causing the associated 
earthquake. We will also perform experiments to assess the effect of localized assimilation and the placement of measurement sensors on the assimilated solution. This is followed by an additional twin experiment that analyzes the effect of initial ensemble generation and the ensemble size on assimilation runs. Further, we also validate our results using real gauge data of the actual event. In the process we will demonstrate the efficacy of assimilation with AMR to obtain improved state estimates and the corresponding uncertainty.

\subsection{Model parameters}

For the tsunami simulation, the computational domain is the South Pacific ocean off the Chilean coast shown in Figure 6. The domain contains islands/features such as Easter Island and the Galapagos Islands. In the coarsest mesh, these features are not visible. During the simulation, as the mesh refines, the features become more apparent. The model parameters, including the mesh parameters, time stepping parameters, and topography are given in Table 1 . The marked points 15, 12 and 32412 represent locations where the quantities of interest, such as water surface elevation, are recorded. In Figure 6, the two black lines given by the horizontal line at -30 and the vertical line at -90 , represent the partition of the domain at the coarsest grid level into 4 patches. This is related to the specifics of the GEOCLAW software.

Table 1: Model parameters for simulating Chile tsunami event of 2010

\begin{tabular}{ll}
\hline Computational domain & $x \in\left[-120^{\circ},-60^{\circ}\right]$ and $y \in\left[-60^{\circ}, 0^{\circ}\right]$ \\
Coarsest discretization & 100 cells $\times 100$ cells \\
Simulation time & 13 hours \\
Time stepping & Initial time step $=0.016$ seconds and desired $c f l=0.75$ \\
Topography & 10 minute ETOPO2 from NGDC 2 \\
\hline
\end{tabular}

\footnotetext{
${ }^{2}$ National geophysical data center (NGDC) GEODAS grid translator 2010
} 
Table 2: Topography parameters for ensemble generation and twin experiment

\begin{tabular}{|c|c|c|c|c|c|c|c|c|c|}
\hline Statistic & Strike & Length & Width & Depth & Slip & Rake & Dip & Longitude & Latitude \\
\hline Truth $^{*}$ & 16.0 & 450.0 & 100.0 & 35.0 & 15.0 & 104.0 & 14.0 & -72.668 & -35.826 \\
\hline Biased $^{*}$ & - "- & -" - & -" - & -" - & 35.0 & -" - & -" - & -" - & -" - \\
\hline
\end{tabular}

${ }^{*}$ Mean topography. Units [meter, kilometer, kilometer, kilometer, meter, meter, meter $\left.{ }^{\circ},{ }^{\circ}\right]$

\subsection{Reference solution}

The reference solution is simulated using the "true" fault parameters of the Okada model, given in Table 2 that produces the associated slip in the fault region and the sea floor deformation shown in Figure 7 a The bathymetry change causes a tsunami with the water surface elevation snapshots given in Figure $8 \mathrm{a}$. The figures show a primary wave traveling in the north-west direction. At approximately 4.0 hours, a section of the wave traveling along the north, reflects off the coast of Peru to form a secondary wave traveling in the south-west direction.

\subsection{Twin experiment}

In this experiment, we set up a model run with biased fault parameters in comparison to the truth. The twin experiment is aimed to demonstrate that the run with the biased fault parameters can be effectively assimilated using the measurements from the "true" solution.

\subsubsection{Biased run}

The bias in the fault parameters given in Table 2 is a slip of 35.0 meters compared to 15.0 meters for the truth. The additional slip in the fault regions relates to a larger bathymetry change shown in Figure $7 \mathrm{~b}$, leading to a stronger tsunami. This can be seen in the snapshots in Figure $8 b$ for a free run under this biased initial condition. Note that free run means a model run that is not assimilated. Comparing the "true" state in Figure $8 \mathrm{a}$ with the free run state in Figure $8 \mathrm{~b}$, the tsunami generated from the biased fault parameters has a larger amplitude than the true state, till the waves exit the computational domain. 


\subsubsection{Initial ensemble generation}

To generate the initial ensemble of states, we implement the algorithm discussed in Section 5. We treat the biased slip fault parameter as the mean slip for the free run. We take a sample of $100\left(N_{s}\right)$ slips assuming that the slip follows a normal distribution of mean 35.0 meters and a standard deviation of 4 meters. Figure 9 shows the sample distribution of the slips generated with a mean of 20.0 meters added to the true slip of 15.0 meters. A forward integration of the model until the checkpoint time $\left(t_{c p}\right)$ of 2.0 hours, with each unique fault parameter as an initial condition, results in the subspace of tsunamis. Proceeding with a singular value decomposition of the covariance matrix, followed by the construction of the perturbation matrix, we generate the initial ensemble of desired size. For illustration, Figure 10 is the mean water surface elevation at the checkpoint time $\left(t_{c p}\right)$ of 2.0 hours for the model run simulated using the biased fault parameter as the initial condition. Figure 11 shows the first 9 members of the 16 ensembles $\left(n_{s}\right)$ that are generated after using the initial ensemble generation algorithm. At the checkpoint time $\left(t_{c p}\right)$ of 2.0 hours, we resume the simulation, while assimilating the ensemble members with the measurement data from the "true" solution.

For all our experiments,we have applied ESTKF to assimilate the synthetic data every 15 minutes, setting the inflation factor to 1.1. We assess the assimilated water surface elevation, in comparison to the model free run (non-assimilative run). The error norm used is,

$$
\|e(t)\|_{2}=\left\|\psi_{\text {true }}(t)-\hat{\psi}(t)\right\|_{2}
$$

where $\hat{\psi}$ can be the assimilated state or the free run state.

\subsubsection{Experiment 1: Effect of observation configuration and localization radius}

In this experiment, we analyze the effect of the number of measurements and localization radius on the assimilated state estimates. We have chosen various observation grids ranging from the region containing 4 (a sparse network), 12, 35 and 42 (a dense network) sensors, distributed in the Pacific ocean as shown in Figure 12 For each of the observation grids, we use $16\left(n_{s}\right)$ ensemble members with localization radius of $3^{\circ}$, $5^{\circ}, 10^{\circ}, 15^{\circ}, 20^{\circ}, 25^{\circ}$ and $35^{\circ}$, along with a global assimilation. The observation error 
is assumed to be normally distributed with the standard deviation of observation error set to 0.005 meters. At every assimilation step, the state estimate after the assimilation is compared to the truth and the errors are calculated.

At the fault region, varying slip parameters for the truth and the biased model runs leads to a differing bathymetry profile. For error calculation for this experiment, we omit the region around the fault, that is $\left(-76.0^{\circ},-68.0^{\circ}\right) \times\left(-40.0^{\circ},-32.0^{\circ}\right)$. The error plots for all the mentioned observation configurations are given in Figure 13 . It was found that the error plots produced without ignoring the fault region give a similar trend as the plots in Figure 13 . However, ignoring the fault region makes the trend more pronounced. The figures plot the difference between the free run and the truth, the difference between the globally assimilated state estimate and the truth, and the difference between the locally assimilated state estimate and the truth for various localization radii.

Figure 13 indicates that irrespective of the observation configuration, as the localization radius increases, the estimated error between the "true" solution and the assimilated state decreases till a critical localization radius. Assimilation with further increase in the localization radius (continuing towards a global assimilation) results in errors between assimilation run and the truth being greater than the error between free run and the truth, implying that assimilation starts to degrade the solution and performs worse than the case when the state was not being assimilated. Also, even with a small ensemble of 16 members $\left(n_{s}\right)$, for the assimilation runs with localization radius less than the critical radius, the assimilation run estimates have smaller error compared to the error between non-assimilative free run and the truth, until at least approximately 30 assimilation steps, equal to 450 minutes (or 7.5 hours) of simulation. This corresponds to the time period of 2 hours to 9.5 hours, which may be critical for evacuation measures. For instance, the preparation time required for incoming tsunami at Hawaii is around 10 hours when the tsunami is generated from Chile and 4 hours when the tsunamis is generated from Alask 3 Hence, assimilation proves to be effective during this critical time by providing improved estimate of the state.

\footnotetext{
${ }^{3}$ http://ptwc.weather.gov/hawaii.php
} 
It is found that after 10 hours of simulation, the error norms are practically the same, whether data are assimilated or not. That is the assimilated run converges to the free run. This can be clearly observed in the assimilation of 42 observations in Figure 13d This is because, after around 10 hours, the tsunami wave front has traveled across the computational domain, leaving behind no significant wave fronts in the region. However, in assimilation of other observation grids, the errors seem to increase after nearly 30 assimilation steps. This is attributed to multiple factors. One of the reasons is that a smaller localization radius does not cover the regions near the coast and some small errors persist. The assimilation with 42 observation grids witnessed local assimilation of the coastal regions as well and this reduced the error further. Thus, for this setup, it is found that "small" localization radius with measurements closer to the coastal areas can prove to be effective for assimilation. Increased errors near the end of the simulation can also be attributed to errors due to small ensemble size and ensemble variance inflation at every assimilation step. In Section 6.3.4, we will demonstrate that by taking larger ensemble size, this error can further be reduced.

For the observation configuration with $4,12,35$ and 42 observations, the critical localization radius is approximately $25^{\circ}$ (approximately 2500 kilometers of land), $15^{\circ}$ (approximately 1500 kilometers of land), $15^{\circ}$ and $15^{\circ}$, respectively. The state estimate for each of the assimilation runs with the critical localization radius is given in Figures $14 \mathrm{a}, 14 \mathrm{~b}, 14 \mathrm{c}$ and $14 \mathrm{~d}$ respectively. From the state estimates with assimilation using observation configuration of 4 sensors and 12 sensors (Figure 14a and $14 \mathrm{~b}$, at 5.0 and 6.0 hours, the tsunami wavefront has smaller estimated water surface elevation (amplitude) when assimilation is performed with 12 observations, when compared to assimilation with 4 observations. The smaller amplitude conforms to the truth at the time of 5.0 and 6.0 hours in Figure $8 \mathrm{a}$ This suggests that in this particular experiment, assimilating with 12 observations is more effective than using 4 observations. We further compare the filter state estimate (Figure 14b and 14c) obtained by assimilating observations with configuration of 12 sensors to the filter state estimate obtained by assimilating observations with the configuration of 35 sensors. The observation configuration with 35 sensors has more sensors within the same area compared to the observation configuration of 12 sensors. Further, the edge that connects the rightmost 
sensors is equally away from the coast for both the configurations. It is observed that there is not much improvement in the estimated state, even though more observations are assimilated. The observation configuration with 42 sensors has 7 additional sensors to the right, compared to the 35 observation configuration. That is, the edge joining the rightmost sensors for observation configuration with 42 sensors are closer to the coast compared to the observation configuration of 35 sensors. Comparing water surface elevation snapshots at around time of 4.0,5.0 hours in Figure $14 \mathrm{~b}$ and $14 \mathrm{c}$, assimilation using 42 sensors results in waves with higher estimated water surface elevation traveling along the coast when compared to assimilation with 35 observations. This reaffirms the fact that assimilation of sensor data closer to the coast may be helpful.

In summary, this experiment illustrates that the assimilation runs do not always guarantee that the analysis state will have lesser errors than the free run solution. In fact, in this experiment, the assimilation runs with high localization radius perform worse than the free run. One of the possible reasons might be that with high localization radius, the analysis step updates the state vector unreasonably in the regions that are physically far from the observation coordinates. Other reasons include existence of various confounding variables such as ensemble size, observation configuration, standard deviation of observation standard error and most importantly the difference of the initial condition and the truth. To get insights into this phenomenon, we have analyzed assimilation run with various localization radii, while keeping the above-mentioned variables fixed. It is found there exists an optimum localization radius that guarantees that the assimilation is effective. Thus, this experiment of assessing the optimum localization radius serves as a method for model calibration.

\subsubsection{Experiment 2: Effect of initial ensemble generation}

In the previous experiment, the initial ensemble was generated via perturbations in the slip parameter only. Further, though the prior slip information of 35.0 meters was significantly away from the true slip of 15.0 meters, the above experiment demonstrated the effectiveness of assimilation. This experiment differs in a few aspects - 1) the initial ensemble is generated with perturbations in all the fault parameters, 2) the observation grid chosen is 142 sensors in Figure 15. covering regions that are closer to the shore, 
3) the synthetic observations are generated by a model run that takes a random sample of fault parameters from the multivariate Gaussian distribution assuming the mean of the fault parameters is given by $\mu_{\text {topo }}$ in Table 3 and covariance matrix is diagonal with the diagonal entries given by $\left.\sigma_{1}^{2}, 4\right)$ the sample of $100\left(N_{s}\right)$ fault configurations for the initial ensemble generation algorithm assumes a distribution of fault parameters with mean parameters given by $\mu_{\text {topo }}$ in Table 3 and variance terms of the parameters given by $\left.\sigma_{2}^{2}, 5\right)$ the observation error is assumed to be normally distributed with the standard deviation of observation error set to $0.001 \mathrm{~m}$.

Table 3: Topography parameters for ensemble generation for twin experiment 2

\begin{tabular}{cccccccccc}
\hline Statistic & Strike & Length & Width & Depth & Slip & Rake & Dip & Longitude & Latitude \\
\hline$\mu_{\text {topo }}{ }^{\mathrm{a}}$ & 16.0 & 450.0 & 100.0 & 35.0 & 15.0 & 104.0 & 14.0 & -72.668 & -35.826 \\
$\sigma_{1}^{2} \mathrm{~b}$ & 4.0 & 150.0 & 50.0 & 10.0 & 5.0 & 20.0 & 4.0 & 3.0 & 3.0 \\
$\sigma_{2}^{2} \mathrm{c}$ & 1.6 & 45.0 & 10.0 & 3.5 & 1.5 & 10.4 & 1.4 & 2.0 & 2.0 \\
\hline
\end{tabular}

${ }^{a}$ Mean topography. Units [meter, kilometer, kilometer, kilometer, meter, meter, meter $\left.,^{\circ},^{\circ}\right]$

${ }^{\mathrm{b}}$ Variance for generating initial ensemble for twin experiment $\left(\right.$ Units $\left.^{2}\right)$

${ }^{\mathrm{c}}$ Variance for generating synthetic observations $\left(\right.$ Units $\left.^{2}\right)$

We can analyze the effect of perturbing all the fault parameters in the process of generating the initial ensemble mentioned in Section 5 Figure 16 plots the eigen decomposition of the covariance matrix of $100\left(N_{s}\right)$ model states, with the red line indicating the cumulative percentage variance explained by the eigen modes. From the figure, it can be seen that the first 10 eigenvalues explain more than $85 \%$ of the total variance. We can also analyze the effect of the sample size on the percentage variance explained by the eigen modes in Table 4. The table suggests that as the sample size increases, a higher number of eigen modes account for the unique variance among the states. It is found that the subspace of the initial ensemble that can be generated is richer when compared to just perturbing the slip as in twin experiment 1 . 
Table 4: Percentage captured variance with varying ensemble size in initial ensemble generation in twin experiment 2 of the Chile tsunami simulation

\begin{tabular}{cccccccccccccc}
\hline \multirow{2}{*}{ Ensemble } & \multicolumn{11}{c}{ \% explained variance by eigen modes } \\
\cline { 2 - 13 } & 1 & 2 & 3 & 4 & 5 & 6 & 7 & 8 & 9 & 10 & 11 & 12 & Total \\
\hline 16 & 32 & 21 & 12 & 10 & 7 & 5 & 3 & 3 & 1 & 0 & 0 & 0 & 100 \\
32 & 27 & 18 & 11 & 9 & 7 & 6 & 4 & 3 & 2 & 2 & 1 & 1 & 91 \\
40 & 27 & 19 & 10 & 8 & 7 & 6 & 4 & 3 & 2 & 2 & 1 & 1 & 90 \\
64 & 24 & 18 & 10 & 8 & 6 & 6 & 4 & 3 & 2 & 2 & 1 & 1 & 85 \\
80 & 24 & 17 & 10 & 7 & 6 & 5 & 4 & 4 & 3 & 2 & 2 & 1 & 85 \\
100 & 24 & 16 & 10 & 7 & 6 & 5 & 4 & 4 & 3 & 2 & 2 & 1 & 84 \\
\hline
\end{tabular}

Since we have taken a reasonably dense observation network, with observations covering near the coastal regions, the error calculations need not ignore the fault regions. Moreover, it is found that lower observation standard deviation of 0.001 meters gives reasonably good performance for the global assimilation as well. However, this is subjective to various factors defining the test setup such as the initial conditions, observation network etc. Figure 17a plots the difference between the "true" state and free run (non-assimilative run) state, and the difference between the "true" state and the globally assimilated state estimate for various ensemble sizes at the assimilation times. By analyzing the effect of ensemble size, it is found that an assimilation run with 64 members consistently produces more accurate state estimates than the non-assimilative free run. Further, as the ensemble size increases, the error decreases. It is found that the number of members required depends on how far the initial ensemble is from the truth. That is, by repeating the experiment with different sets of synthetic observations, it is found that in some cases assimilation run with just 32 ensemble members are sufficient to produce consistently less error than the free run. This is tested by another set of synthetic measurements obtained from a model run that uses the topography parameters sampled from a multivariate normal distribution with mean and variance as $\mu_{\text {topo }}$ and $\sigma_{2}^{2}$ respectively in Table 3 . Assimilation is performed using the same initial ensemble that was generated earlier. From Figure $17 \mathrm{~b}$, assimilating the new measurement values 
results with 32 ensemble members providing consistently smaller errors than the free run. This suggests that the minimum ensemble size required for effective assimilation depends upon how far the first guess of the initial condition is from the truth.

For the case just described (Figure 17b), Figure 18a shows the estimated state at various assimilation steps and Figure $18 \mathrm{~b}$ shows that over the simulation time, successive assimilations reduce the root mean square in the water surface elevation. This translates to the fact that with every assimilation step, we obtain state estimates with better certainty. Also, some uncertainty persists near the fault regions that decreases with further assimilation steps.

\subsection{Real gauge experiment}

The DART buoys measure the pressure at the sea floor in deep water to provide an early indication of the magnitude of a tsunami. Gauge data of the real Chile 2010 event is available via DART (Deep-ocean Assessment and Reporting of Tsunamis) buoy $32412^{4}$ For this experiment, we will illustrate data assimilation using 2 levels. Also, we will use observation localization with support radius $20^{\circ} \approx 2000 \mathrm{~km}$.

The standard deviation of observation error is set to 0.005 meters. Assimilation is done every 15 minutes with $16\left(n_{s}\right)$ ensemble members and the inflation factor is set to 1.1. Figure 19 compares the assimilation mean state (right side) to the free run state (left side). The rectangular boxes in the snapshot represent refined regions by a factor of 2 in both directions. The figures show that the amplitude of the tsunami wavefront propagating in the west direction is reduced when the gauge data is taken into account. Also, since the assimilation is done on a supermesh, there are more level 2 regions in the assimilated state than the corresponding free run state. This is expected because at the assimilation step, refinement is performed in regions where at least one of the ensemble members has a level 2 region at the end of its forecast step. At time of 5.0 hours, unlike the free run, the best estimate of the water surface elevation snapshot for assimilation run reveals a series of waves traveling in south-east direction towards the coast. This may have implications that data assimilation is effective at capturing waves

4 http://www.ndbc.noaa.gov/dart.shtml 
that might otherwise have not been resolved in non-assimilative simulations. Further, the AMR capability captures these waves and refines along the regions until it reaches the coast. We observe that during the entire simulation, more refined regions are automatically obtained along the coast when assimilation is performed, in comparison to the non-assimilative free run. Thus, the AMR is effective in capturing the oscillations created by the state update. Most importantly, Figure 20 outlines the effect of assimilation through the gauge output for the free run and simulation with assimilation. With assimilation, the gauge output (bottom) closely follows the real observation data. This was not possible otherwise.

\section{Summary and conclusions}

We have used ensemble Kalman filter based data assimilation to demonstrate global assimilation and local assimilation on a numerical model with multi-level adaptive mesh refinement. Specifically, we have developed a data assimilation framework by coupling the GEOCLAW model with the data assimilation library PDAF. A strength of the research presented, is the fact that GEOCLAW and PDAF are open software. Further, we demonstrated the use of a supermesh technique to tackle the situation of ensemble members having different mesh structure. For data assimilation of tsunami models, we have outlined an algorithm to generate the initial ensemble state and performed various twin experiments assessing the effect of observation configuration with respect to number of observations, localization radius and ensemble size on errors between assimilated state and free run. This indicates that assimilation is effective. Additionally, we showed that more accurate state estimates can be obtained while reducing uncertainty with assimilation steps. We have further provided the assimilation of actual gauge data for the Chile earthquake of Feb 27, 2010. We concurrently showed the execution of assimilation with adaptive mesh refinement up to 2 levels. We demonstrated that the AMR capability captured the oscillations in the assimilated state and tracked the waves as they traveled towards the coast. This was reaffirmed with the estimated water surface elevation closely following the true values at the DART gauge. The AMR-coupled data assimilation framework has been tested with 
higher refinement levels (of up to 5). The results presented in this paper are generated from the code for GEOCLAW at https://zenodo.org/badge/latestdoi/ 30735579 and the code for AMRCLAW at https://zenodo.org/badge/ latest doi/92109632. Research is underway to develop test cases that can benchmark the efficacy of the AMR with ensemble data assimilation. Even though the preliminary results are applied on a tsunami model, other applications being considered are assimilation of hurricane storm surges as well.

The study is aimed to provide some primary insights in the development of data driven computational framework using AMR capable numerical models. In particular, ensemble data assimilation requires integration of several model runs, This can be computationally expensive for large scale hydrodynamic models. On the contrary, the numerical codes with AMR technique offer advantages with respect to computational speed as areas that are not of interest such as the deep ocean can retain coarse mesh. Hence, ensemble data assimilation with AMR is ideal for use in real-time forecasting scenarios, where time of simulations is of essence and may serve as a bottleneck for initiating evacuation measures. The coupling methodology demonstrated in this study, can be extended to various models currently in use. The possible areas to further explore are storm surges and wave runup. Research is underway to test the developed capability with other historical events. This will aid in the development of a potential real-time forecasting tool, that offers computational speed and uncertainty quantification simultaneously.

\section{Acknowledgments}

The authors acknowledge the support of the King Abdullah University of Science and Technology Competitive Research Grant Program, award number OCRF-2014CRG3-62140389/ORS\#2156.

\section{References}

[1] Deborah Balk, Mark R Montgomery, Gordon McGranahan, Donghwan Kim, Valentina Mara, Megan Todd, Thomas Buettner, and Audrey Dorélien. Map- 
ping urban settlements and the risks of climate change in Africa, Asia and South America. Population dynamics and climate change, 80, 2009.

[2] Brent W. Ache, Kristen M. Crossett, Percy A. Pacheco, Jeffery E. Adkins, and Peter C. Wiley. "The coast" is complicated: A model to consistently describe the nation's coastal population. Estuaries and coasts, 38(1):151-155, 2015.

[3] Barbara Neumann, Athanasios T. Vafeidis, Juliane Zimmermann, and Robert J. Nicholls. Future coastal population growth and exposure to sea-level rise and coastal flooding - A global assessment. PLOS ONE, 10(3):1-34, 032015. doi: 10.1371/journal.pone.0118571. URL https://doi.org/10.1371/ journal.pone.0118571

[4] Richard A. Luettich Jr., Johannes J. Westerink, and Norman W. Scheffner. ADCIRC: An advanced three-dimensional circulation model for shelves, coasts, and estuaries. Report 1. Theory and methodology of ADCIRC-2DDI and ADCIRC3DL. Technical report, DTIC Document, 1992.

[5] Hrvoje Jasak, Aleksandar Jemcov, Zeljko Tukovic, et al. OpenFOAM: A C++ library for complex physics simulations. In International workshop on coupled methods in numerical dynamics, volume 1000, pages 1-20. IUC Dubrovnik, Croatia, 2007.

[6] Eric P. Chassignet, Harley E. Hurlburt, Ole Martin Smedstad, George R. Halliwell, Patrick J. Hogan, Alan J. Wallcraft, Remy Baraille, and Rainer Bleck. The \{HYCOM (HYbrid Coordinate Ocean Model) data assimilative system. Journal of Marine Systems, 65(1-4):60 - 83, 2007. ISSN 0924-7963. doi: https://doi. org/10.1016/j.jmarsys.2005.09.016. URL http://www.sciencedirect. com/science/article/pii/s0924796306002855. Marine Environmental Monitoring and PredictionSelected papers from the 36th International Liège Colloquium on Ocean Dynamics36th International Liège Colloquium on Ocean Dynamics.

[7] G. Madec. NEMO ocean engine. Note du Pôle de modélisation, Institut PierreSimon Laplace (IPSL), France, No 27, ISSN No 1288-1619, 2008. 
[8] Zhijin Li, James C. McWilliams, Kayo Ide, and John D. Farrara. A multiscale variational data assimilation scheme: Formulation and illustration. Monthly Weather Review, 143(9):3804-3822, 2015. doi: 10.1175/MWR-D-14-00384.1. URL http://dx.doi.org/10.1175/MWR-D-14-00384.1.

[9] Altuğ Aksoy, David C. Dowell, and Chris Snyder. A multicase comparative assessment of the ensemble Kalman filter for assimilation of radar observations. Part I: Storm-scale analyses. Monthly Weather Review, 137(6):1805-1824, 2009. doi: 10.1175/2008MWR2691.1. URL http://dx.doi.org/10.1175/ 2008MWR2691.1.

[10] Jason T. Holt, J. Icarus Allen, Roger Proctor, and Francis Gilbert. Error quantification of a high-resolution coupled hydrodynamic-ecosystem coastal-ocean model: Part 1 model overview and assessment of the hydrodynamics . Journal of Marine Systems, 57(1-2):167 - 188, 2005. ISSN 0924-7963. doi: https://doi. org/10.1016/j.jmarsys.2005.04.008. URL http://www. sciencedirect. com/science/article/pii/s0924796305001053.

[11] J. I. Allen, P. J. Somerfield, and F. J. Gilbert. Quantifying uncertainty in high-resolution coupled hydrodynamic-ecosystem models. Journal of Marine Systems, 64(1-4):3 - 14, 2007. ISSN 0924-7963. doi: https://doi.org/10. 1016/j.jmarsys.2006.02.010. URL http://www.sciencedirect.com/ science/article/pii/S0924796306001035. Contributions from Advances in Marine Ecosystem Modelling Research, 27-29 June, 2005, Plymouth, \{UKAMEMR .

[12] James D. Brown, Tom Spencer, and Iris Moeller. Modeling storm surge flooding of an urban area with particular reference to modeling uncertainties: A case study of Canvey Island, United Kingdom. Water Resources Research, 43 (6), 2007. ISSN 1944-7973. doi: 10.1029/2005WR004597. URL http: //dx.doi.org/10.1029/2005WR004597. W06402.

[13] P. Courtier, E. Andersson, W. Heckley, D. Vasiljevic, M. Hamrud, A. Hollingsworth, F. Rabier, M. Fisher, and J. Pailleux. The ECMWF imple- 
mentation of three-dimensional variational assimilation (3D-Var). I: Formulation. Quarterly Journal of the Royal Meteorological Society, 124(550):17831807, 1998. ISSN 1477-870X. doi: 10.1002/qj.49712455002. URL http: //dx.doi.org/10.1002/qj.49712455002.

[14] P. Courtier, J.-N. Thépaut, and A. Hollingsworth. A strategy for operational implementation of 4D-Var, using an incremental approach. Quarterly Journal of the Royal Meteorological Society, 120(519):1367-1387, 1994. ISSN 1477-870X. doi: 10.1002/qj.49712051912. URL http://dx.doi.org/10.1002/qj. 49712051912 .

[15] Rudolph Emil Kalman. A new approach to linear filtering and prediction problems. Journal of basic Engineering, 82(1):35-45, 1960.

[16] Rudolph E. Kalman and Richard S. Bucy. New results in linear filtering and prediction theory. Journal of basic engineering, 83(3):95-108, 1961.

[17] Gerald L Smith, Stanley F Schmidt, and Leonard A McGee. Application of statistical filter theory to the optimal estimation of position and velocity on board a circumlunar vehicle. National Aeronautics and Space Administration, 1962.

[18] Ibrahim Hoteit, George Korres, and George Triantafyllou. Comparison of extended and ensemble based Kalman filters with low and high resolution primitive equation ocean models. Nonlinear Processes in Geophysics, 12(5):755765, 2005. doi: 10.5194/npg-12-755-2005. URL http://www . nonlinprocesses-geophys.net/12/755/2005/

[19] Geir Evensen. Sequential data assimilation with a nonlinear quasi-geostrophic model using Monte Carlo methods to forecast error statistics. Journal of Geophysical Research: Oceans, 99(C5):10143-10162, 1994. ISSN 2156-2202. doi: 10.1029/94JC00572. URL http://dx.doi.org/10.1029/94JC00572

[20] Geir Evensen. The Ensemble Kalman Filter: :Theoretical formulation and practical implementation. Ocean Dynamics, 53(4):343-367, 2003. ISSN 1616-7228. 
doi: 10.1007/s10236-003-0036-9. URL http://dx.doi.org/10.1007/ s10236-003-0036-9.

[21] Jan Mandel, Loren Cobb, and Jonathan D. Beezley. On the convergence of the Ensemble Kalman filter. Applications of Mathematics, 56(6):533-541, Dec 2011. ISSN 1572-9109. doi: 10.1007/s10492-011-0031-2. URL https://doi. org/10.1007/s10492-011-0031-2

[22] Knut Arild Lisæter, Julia Rosanova, and Geir Evensen. Assimilation of ice concentration in a coupled ice-ocean model, using the Ensemble Kalman filter. Ocean Dynamics, 53(4):368-388, 2003. ISSN 1616-7228. doi: 10.1007/ s10236-003-0049-4. URL http://dx.doi.org/10.1007/s10236003-0049-4.

[23] Chris Snyder and Fuqing Zhang. Assimilation of simulated Doppler radar observations with an Ensemble Kalman filter. Monthly Weather Review, 131(8): 1663-1677, 2003. doi: 10.1175//2555.1. URL http://dx.doi.org/10. $1175 / / 2555.1$

[24] Georg A. Grell and Dezsô Dévényi. A generalized approach to parameterizing convection combining ensemble and data assimilation techniques. Geophysical Research Letters, 29(14):38-1-38-4, 2002. ISSN 1944-8007. doi: 10.1029/ 2002GL015311. URL http://dx.doi.org/10.1029/2002GL015311.

[25] Talea Mayo, Troy Butler, Clint Dawson, and Ibrahim Hoteit. Data assimilation within the Advanced Circulation (ADCIRC) modeling framework for the estimation of Manning's friction coefficient . Ocean Modelling, 76:43 58, 2014. ISSN 1463-5003. doi: https://doi.org/10.1016/j.ocemod.2014.01. 001. URL http://www.sciencedirect.com/science/article/ pii/S146350031400002X.

[26] T. Butler, M. U. Altaf, C. Dawson, I. Hoteit, X. Luo, and T. Mayo. Data Assimilation within the Advanced Circulation (ADCIRC) Modeling Framework for Hurricane Storm Surge Forecasting. Monthly Weather Review, 140(7):2215-2231, 
2012. doi: 10.1175/MWR-D-11-00118.1. URL http://dx.doi.org/10. $1175 /$ MWR-D-11-00118.1.

[27] G. Triantafyllou, I. Hoteit, X. Luo, K. Tsiaras, and G. Petihakis. Assessing a robust ensemble-based Kalman filter for efficient ecosystem data assimilation of the Cretan Sea. Journal of Marine Systems, 125:90 - 100, 2013. ISSN 0924-7963. doi: http://dx.doi.org/10.1016/j.jmarsys.2012.12. 006. URL http://www.sciencedirect.com/science/article/ pii/S0924796312002436. Advances in Marine Ecosystem Modelling Research III.

[28] Thomas M. Hamill. Ensemble-based atmospheric data assimilation. Predictability of weather and climate, pages 124-156, 2006.

[29] Martin Ehrendorfer. A review of issues in ensemble-based Kalman filtering. Meteorologische Zeitschrift, 16(6):795-818, 12 2007. doi: 10.1127/0941-2948/

a 2007/0256. URL http://dx.doi.org/10.1127/0941-2948/2007/ 0256.

[30] P. L. Houtekamer and Fuqing Zhang. Review of the Ensemble Kalman filter for atmospheric data assimilation. Monthly Weather Review, 144(12):4489-4532,

2016. doi: 10.1175/MWR-D-15-0440.1. URL http://dx.doi.org/10 . $1175 / M W R-D-15-0440.1$.

[31] Christopher A. Edwards, Andrew M. Moore, Ibrahim Hoteit, and Bruce D. Cornuelle. Regional ocean data assimilation. Annual review of marine science, 7: 21-42, 2015.

[32] R. N. Bannister. A review of forecast error covariance statistics in atmospheric variational data assimilation. I: Characteristics and measurements of forecast error covariances. Quarterly Journal of the Royal Meteorological Society, 134 (637):1951-1970, 2008. ISSN 1477-870X. doi: 10.1002/qj.339. URL http: //dx.doi.org/10.1002/qj.339 
[33] A. R. Robinson, P. F. J. Lermusiaux, and N. Q. Sloan. Data assimilation. The sea, 10:541-594, 1998.

[34] Jeffrey S. Whitaker and Thomas M. Hamill. Ensemble data assimilation without perturbed observations. Monthly Weather Review, 130(7):19131924, 2002. doi: 10.1175/1520-0493(2002)130<1913:EDAWPO>2.0.CO;2. URL http://dx.doi.org/10.1175/1520-0493(2002) 130<1913: EDAWPO $>2.0 . \mathrm{CO} ; 2$

[35] Jeffrey L. Anderson. An Ensemble Adjustment Kalman Filter for Data Assimilation. Monthly Weather Review, 129(12):2884-2903, 2001. doi: 10.1175/15200493(2001)129<2884:AEAKFF>2.0.CO;2. URL http://dx.doi.org/ $10.1175 / 1520-0493(2001) 129<2884: \mathrm{AEAKFF}>2.0 . \mathrm{CO} ; 2$.

[36] Jeffrey Anderson, Tim Hoar, Kevin Raeder, Hui Liu, Nancy Collins, Ryan Torn, and Avelino Avellano. The Data assimilation Research Testbed: A community facility. Bulletin of the American Meteorological Society, 90(9):1283-1296, 2009.

doi: 10.1175/2009BAMS2618.1. URL http://dx.doi.org/10.1175/ 2009BAMS2618.1.

[37] Dinh Tuan Pham. Stochastic methods for sequential data assimilation in strongly nonlinear systems. Monthly Weather Review, 129(5):11941207, 2001. doi: 10.1175/1520-0493(2001)129<1194:SMFSDA>2.0.CO;2. URL http://dx.doi.org/10.1175/1520-0493(2001)129<1194: SMFSDA $>2.0 . \mathrm{CO} ; 2$.

[38] Ibrahim Hoteit, Dinh-Tuan Pham, and Jacques Blum. A simplified reduced order Kalman filtering and application to altimetric data assimilation in Tropical Pacific. Journal of Marine Systems, 36(1-2):101 - 127, 2002. ISSN 0924-7963. doi: https://doi.org/10.1016/S0924-7963(02)00129X. URL http://www.sciencedirect.com/science/article/ pii/s092479630200129X.

[39] Craig H. Bishop, Brian J. Etherton, and Sharanya J. Majumdar. Adaptive sampling with the Ensemble Transform Kalman Filter. Part I: Theoretical aspects. 
Monthly Weather Review, 129(3):420-436, 2001. doi: 10.1175/1520-0493(2001) 129<0420:ASWTET>2.0.CO;2. URL http://dx.doi.org/10.1175/ 1520-0493(2001)129<0420:ASWTET>2.0.CO;2.

[40] Dinh Tuan Pham, Jacques Verron, and Marie Christine Roubaud. A singular evolutive extended Kalman filter for data assimilation in oceanography. Journal of Marine Systems, 16(3):323 - 340, 1998. ISSN 0924-7963. doi: http://dx.doi.org/ 10.1016/S0924-7963(97)00109-7. URL http://www.sciencedirect. com/science/article/pii/s0924796397001097.

[41] V. Carmillet, J.-M. Brankart, P. Brasseur, H. Drange, G. Evensen, and J. Verron. A singular evolutive extended Kalman filter to assimilate ocean color data in a coupled physical-biochemical model of the North Atlantic ocean. Ocean Modelling, 3(3):167 - 192, 2001. ISSN 1463-5003. doi: http://dx.doi.org/10.1016/S14635003(01)00007-5. URL http://www.sciencedirect.com/science/ article/pii/S1463500301000075

[42] A. W. Heemink, M. Verlaan, and A. J. Segers. Variance reduced Ensemble Kalman filtering. Monthly Weather Review, 129(7):17181728, 2001. doi: 10.1175/1520-0493(2001)129<1718:VREKF>2.0.CO;2. URL http://dx.doi.org/10.1175/1520-0493(2001)129<1718: VREKF $>2.0 . \mathrm{CO} ; 2$,

[43] Lars Nerger, Tijana Janjić, Jens Schröter, and Wolfgang Hiller. A unification of Ensemble square root Kalman filters. Monthly Weather Review, 140(7):23352345, 2012. doi: 10.1175/MWR-D-11-00102.1. URL http://dx.doi. org/10.1175/MWR-D-11-00102.1.

[44] Jeffrey L. Anderson. A local least squares framework for ensemble filtering. Monthly Weather Review, 131(4):634-642, 2003. doi: 10.1175/15200493(2003)131<0634:ALLSFF>2.0.CO;2. URL http://dx.doi.org/10 . $1175 / 1520-0493(2003) 131<0634:$ ALLSFF $>2.0 . C O ; 2$.

[45] Jeffrey L. Anderson. An adaptive covariance inflation error correction algorithm for ensemble filters. Tellus A, 59(2):210-224, 2007. ISSN 1600-0870. 
doi: 10.1111/j.1600-0870.2006.00216.x. URL http://dx.doi.org/10 . $1111 / j .1600-0870.2006 .00216 . x$

[46] Jeffrey S. Whitaker and Thomas M. Hamill. Evaluating methods to account for system errors in ensemble data assimilation. Monthly Weather Review, 140(9):3078-3089, 2012. doi: 10.1175/MWR-D-11-00276.1. URL http: //dx.doi.org/10.1175/MWR-D-11-00276.1

[47] Xiaodong Luo and Ibrahim Hoteit. Robust ensemble filtering and its relation to covariance inflation in the Ensemble Kalman filter. Monthly Weather Review, 139(12):3938-3953, 2011. doi: 10.1175/MWR-D-10-05068.1. URL http: //dx.doi.org/10.1175/MWR-D-10-05068.1

[48] P. L. Houtekamer and Herschel L. Mitchell. A sequential Ensemble Kalman filter for atmospheric data assimilation. Monthly Weather Review, 129(1): 123-137, 2001. doi: 10.1175/1520-0493(2001)129<0123:ASEKFF>2.0.CO;2. URL http://dx.doi.org/10.1175/1520-0493(2001)129<0123: ASEKFF $>2.0 . \mathrm{CO} ; 2$.

[49] Brian R. Hunt, Eric J. Kostelich, and Istvan Szunyogh. Efficient data assimilation for spatiotemporal chaos: A local ensemble transform Kalman filter. Physica D: Nonlinear Phenomena, 230(1-2):112 - 126, 2007. ISSN 0167-2789. doi: https://doi.org/10.1016/j.physd.2006.11. 008. URL http://www.sciencedirect.com/science/article/ pii/S0167278906004647, Data Assimilation.

[50] Lars Nerger, Tijana Janjić, Jens Schröter, and Wolfgang Hiller. A regulated localization scheme for ensemble-based Kalman filters. Quarterly Journal of the Royal Meteorological Society, 138(664):802-812, 2012. ISSN 1477-870X. doi: 10.1002/qj.945. URL http://dx.doi.org/10.1002/qj.945

[51] Christian L. Keppenne. Data assimilation into a primitive-equation model with a parallel Ensemble Kalman filter. Monthly Weather Review, 128(6):19711981, 2000. doi: 10.1175/1520-0493(2000)128<1971:DAIAPE>2.0.CO;2. 
URL http://dx.doi.org/10.1175/1520-0493(2000)128<1971: DAIAPE $>2.0 . \mathrm{CO} ; 2$

[52] Christian L. Keppenne and Michele M. Rienecker. Initial testing of a massively parallel Ensemble Kalman filter with the Poseidon Isopycnal Ocean General Circulation Model. Monthly Weather Review, 130(12):29512965, 2002. doi: 10.1175/1520-0493(2002)130<2951:ITOAMP>2.0.CO;2. URL http://dx.doi.org/10.1175/1520-0493(2002) 130<2951: ITOAMP $>2 \cdot 0 . \mathrm{CO} ; 2$

[53] Geir Evensen and Peter Jan van Leeuwen. Assimilation of Geosat altimeter data for the Agulhas current using the Ensemble Kalman filter with a quasigeostrophic model. Monthly Weather Review, 124(1):85-96, 1996. doi: 10.1175/15200493(1996)124<0085:AOGADF>2.0.CO;2. URL http://dx.doi.org/ $10.1175 / 1520-0493(1996) 124<0085:$ AOGADF $>2.0 . C O ; 2$.

[54] Andrew C. Lorenc. The potential of the Ensemble Kalman filter for NWP-a comparison with 4D-Var. Quarterly Journal of the Royal Meteorological Society, 129(595):3183-3203, 2003. ISSN 1477-870X. doi: 10.1256/qj.02.132. URL http://dx.doi.org/10.1256/qj.02.132.

[55] Vasily V Titov, Frank I Gonzalez, EN Bernard, Marie C. Eble, Harold O. Mofjeld, Jean C. Newman, and Angie J. Venturato. Real-time tsunami forecasting: Challenges and solutions. In Developing tsunami-resilient communities, pages 41-58. Springer, 2005.

[56] Aditya Riadi Gusman, Anne F. Sheehan, Kenji Satake, Mohammad Heidarzadeh, Iyan Eka Mulia, and Takuto Maeda. Tsunami data assimilation of Cascadia seafloor pressure gauge records from the 2012 Haida Gwaii earthquake. Geophysical Research Letters, 43(9):4189-4196, 2016. ISSN 1944-8007. doi: 10.1002/ 2016GL068368. URL http://dx.doi.org/10.1002/2016GL068368. 2016 GL068368.

[57] Kazuyuki Nakamura, Naoki Hirose, Byung Ho Choi, and Tomoyuki Higuchi. Particle Filtering in Data Assimilation and Its Application to Estimation of 
Boundary Condition of Tsunami Simulation Model, pages 353-366. Springer Berlin Heidelberg, Berlin, Heidelberg, 2009. ISBN 978-3-540-71056-1. doi: 10. 1007/978-3-540-71056-1_19. URL https://doi.org/10.1007/9783-540-71056-1_19.

[58] Kazuyuki Nakamura, Tomoyuki Higuchi, and Naoki Hirose. Sequential data assimilation: Information fusion of a numerical simulation and large scale observation data. J. UCS, 12(6):608-626, 2006.

[59] L. Tang, V. V. Titov, and C. D. Chamberlin. Development, testing, and applications of site-specific tsunami inundation models for real-time forecasting. Journal of Geophysical Research: Oceans, 114(C12), 2009. ISSN 21562202. doi: 10.1029/2009JC005476. URL http://dx.doi.org/10.1029/ 2009JC005476. C12025.

[60] Costas E. Synolakis, Eddie N. Bernard, Vasily V. Titov, Utku Kanoglu, and Frank I. Gonzalez. Validation and verification of tsunami numerical models. Pure and Applied Geophysics, 165(11-12):2197-2228, 2008.

[61] Vasily V. Titov Costas Emmanuel Synolakis. Numerical modeling of tidal wave runup. Journal of Waterway, Port, Coastal, and Ocean Engineering, 124(4):157-171, Issue: object: doi:10.1061/jwped5.1998.124.issue-4, revision: rev:1479482265789-33025:doi:10.1061/jwped5.1998.124.issue4, . doi: 10.1061/(ASCE)0733-950X(1998)124:4(157). URL http:

a //ascelibrary.org/doi/abs/10.1061/\%28ASCE:290733950X281998\%29124\%3A4\%28157\%29.

[62] Ivo Babushka, Jagdish Chandra, and Joseph E. Flaherty. Adaptive computational methods for partial differential equations, volume 16. Siam, 1983.

[63] Randolph E. Bank, Andrew H. Sherman, and Alan Weiser. Some refinement algorithms and data structures for regular local mesh refinement. Sci. Comput. Appl. Math. Comput. Phys. Sci, 1:3-17, 1983. 
[64] Marsha J. Berger and Joseph Oliger. Adaptive mesh refinement for hyperbolic partial differential equations. Journal of Computational Physics, 53(3): 484 - 512, 1984. ISSN 0021-9991. doi: http://dx.doi.org/10.1016/00219991(84)90073-1. URL http://www.sciencedirect.com/science/ article/pii/0021999184900731

[65] Joseph E. Flaherty and Peter K. Moore. Integrated space-time adaptive hprefinement methods for parabolic systems. Applied Numerical Mathematics, 16 (3):317 - 341, 1995. ISSN 0168-9274. doi: http://dx.doi.org/10.1016/01689274(94)00059-P. URL http://wWw.sciencedirect.com/science/ article/pii/016892749400059P

[66] Carsten Burstedde, Lucas C. Wilcox, and Omar Ghattas. p4est: Scalable algorithms for parallel adaptive mesh refinement on forests of octrees. SIAM Journal on Scientific Computing, 33(3):1103-1133, 2011. doi: 10.1137/100791634. URL http://dx.doi.org/10.1137/100791634.

[67] Ralf Deiterding. Detonation Structure Simulation with AMROC, pages 916927. Springer Berlin Heidelberg, Berlin, Heidelberg, 2005. ISBN 978-3-540口 32079-1. doi: 10.1007/11557654_103. URLhttps://doi.org/10.1007/ 11557654_103.

[68] Kyle T. Mandli, Aron J. Ahmadia, Marsha Berger, Donna Calhoun, David L. George, Yiannis Hadjimichael, David I. Ketcheson, Grady I. Lemoine, and Randall J. LeVeque. Clawpack: building an open source ecosystem for solving hyperbolic pdes. PeerJ Computer Science, 2:e68, August 2016. ISSN 2376-5992. doi: 10.7717/peerj-cs.68. URL https://doi.org/10.7717/peerj-cs.68.

[69] M. J. Berger, D. L. George, R. J. LeVeque, and K. T. Mandli. The GeoClaw software for depth-averaged flows with adaptive refinement. Advances in Water Resources, 34:1195-1206, September 2011. doi: 10.1016/j.advwatres.2011.02. 016.

[70] Chiara Piccolo and Mike Cullen. A new implementation of the adaptive mesh transform in the Met office 3D-Var system. Quarterly Journal of the Royal 
Meteorological Society, 138(667):1560-1570, 2012. ISSN 1477-870X. doi: 10.1002/qj.1880. URL http://dx.doi.org/10.1002/qj.1880.

[71] F. Fang, C. C. Pain, I. M. Navon, M. D. Piggott, G. J. Gorman, P. E. Farrell, P. A. Allison, and A. J. H. Goddard. A POD reduced-order 4D-Var adaptive mesh ocean modelling approach. International Journal for Numerical Methods in Fluids, 60(7):709-732, 2009. ISSN 1097-0363. doi: 10.1002/fld.1911. URL http://dx.doi.org/10.1002/fld.1911

[72] Juan Du, Jiang Zhu, Fangxin Fang, C. C. Pain, and I. M. Navon. Ensemble data assimilation applied to an adaptive mesh ocean model. International Journal for Numerical Methods in Fluids, 82(12):997-1009, 2016. ISSN 1097-0363. doi: 10.1002/fld.4247. URL http://dx.doi.org/10.1002/fld.4247. fld. 4247 .

[73] Peter R. Oke and Pavel Sakov. Representation error of oceanic observations for data assimilation. Journal of Atmospheric and Oceanic Technology, 25(6):10041017, 2008.

[74] I. Hoteit, G. Triantafyllou, and G. Korres. Using low-rank Ensemble Kalman filters for data assimilation with high dimensional imperfect models. JNAIAM, 2 (1-2):67-78, 2007.

[75] Randall J. LeVeque. Finite volume methods for hyperbolic problems, volume 31. Cambridge university press, 2002.

[76] Randall J. LeVeque and David L. George. High-resolution finite volume methods for the shallow water equations with bathymetry and dry states. Advanced numerical models for simulating tsunami waves and runup, 10:43-73, 2008.

[77] David L. George. Augmented Riemann solvers for the shallow water equations over variable topography with steady states and inundation. Journal of Computational Physics, 227(6):3089 - 3113, 2008. ISSN 0021-9991. doi: https: //doi.org/10.1016/j.jcp.2007.10.027. URL http://www.sciencedirect. com/science/article/pii/s0021999107004767. 
[78] M. Berger and I. Rigoutsos. An algorithm for point clustering and grid generation. IEEE Transactions on Systems, Man, and Cybernetics, 21(5):1278-1286, Sep 1991. ISSN 0018-9472. doi: 10.1109/21.120081.

[79] Lars Nerger and Wolfgang Hiller. Software for ensemble-based data assimilation systems-Implementation strategies and scalability. Computers \& Geosciences, 55:110 - 118, 2013. ISSN 0098-3004. doi: http://dx.doi.org/ 10.1016/j.cageo.2012.03.026. URL http://www.sciencedirect.com/ science/article/pii/s0098300412001215.

[80] Jérôme Vialard, Frédéric Vitart, Magdalena A. Balmaseda, Timothy N. Stockdale, and David L. T. Anderson. An ensemble generation method for seasonal forecasting with an ocean-atmosphere coupled model. Monthly Weather Review, 133(2):441-453, 2005. doi: 10.1175/MWR-2863.1. URL http: //dx.doi.org/10.1175/MWR-2863.1.

[81] Ibrahim Hoteit, Tim Hoar, Ganesh Gopalakrishnan, Nancy Collins, Jeffrey Anderson, Bruce Cornuelle, Armin Köhl, and Patrick Heimbach. A MITgcm/DART ensemble analysis and prediction system with application to the Gulf of Mexico. Dynamics of Atmospheres and Oceans, 63:1 - 23, 2013. ISSN 0377-0265. doi: http://dx.doi.org/10.1016/j.dynatmoce.2013.03. 002. URL http://www.sciencedirect.com/science/article/ pii/S0377026513000249.

[82] Yoshimitsu Okada. Surface deformation due to shear and tensile faults in a half-space. Bulletin of the Seismological Society of America, 75(4):1135-1154, 1985. URL http://www.bssaonline.org/content/75/4/1135. abstract 


\section{List of Figures}

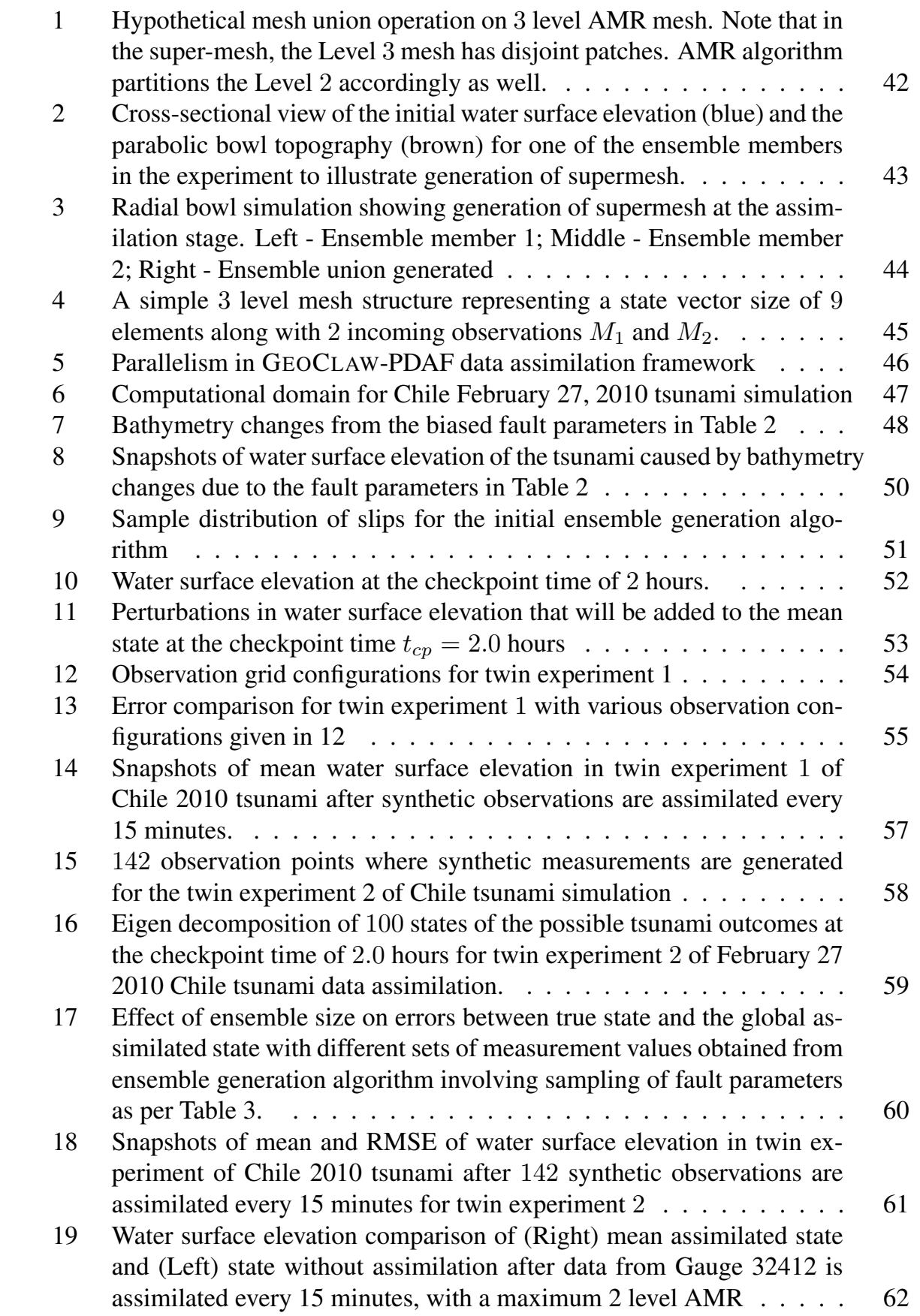


$1013 \quad 20$ (Top) Gauge output for run without assimilation (Bottom) Gauge out-

1014 put for assimilation run of 16 ensemble members with localization ra-

1015

dius of $20^{\circ} \ldots \ldots \ldots \ldots \ldots \ldots$ 


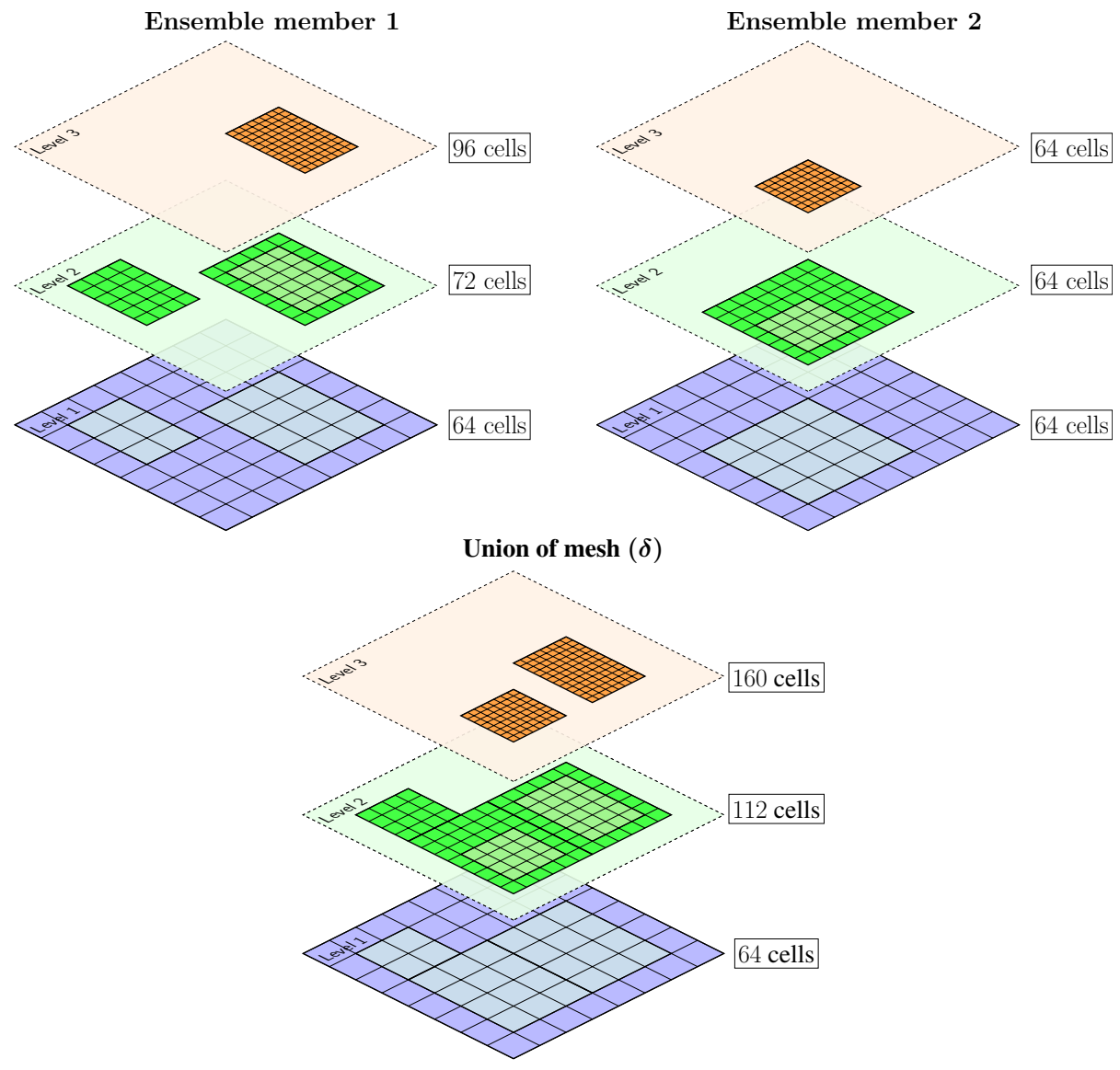

Figure 1: Hypothetical mesh union operation on 3 level AMR mesh. Note that in the super-mesh, the Level 3 mesh has disjoint patches. AMR algorithm partitions the Level 2 accordingly as well. 


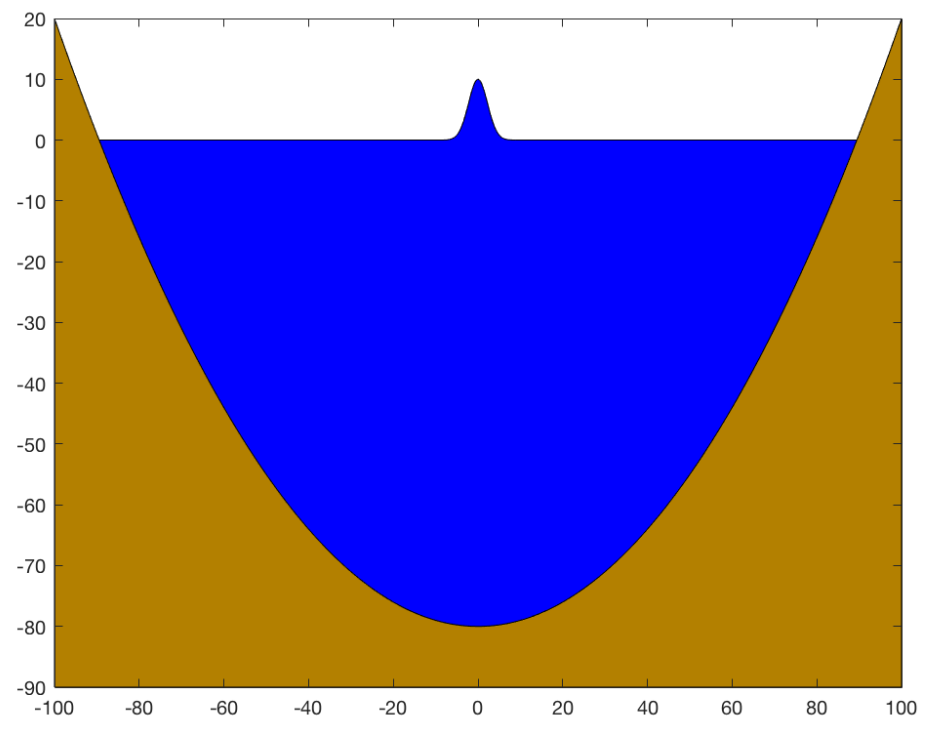

Figure 2: Cross-sectional view of the initial water surface elevation (blue) and the parabolic bowl topography (brown) for one of the ensemble members in the experiment to illustrate generation of supermesh. 

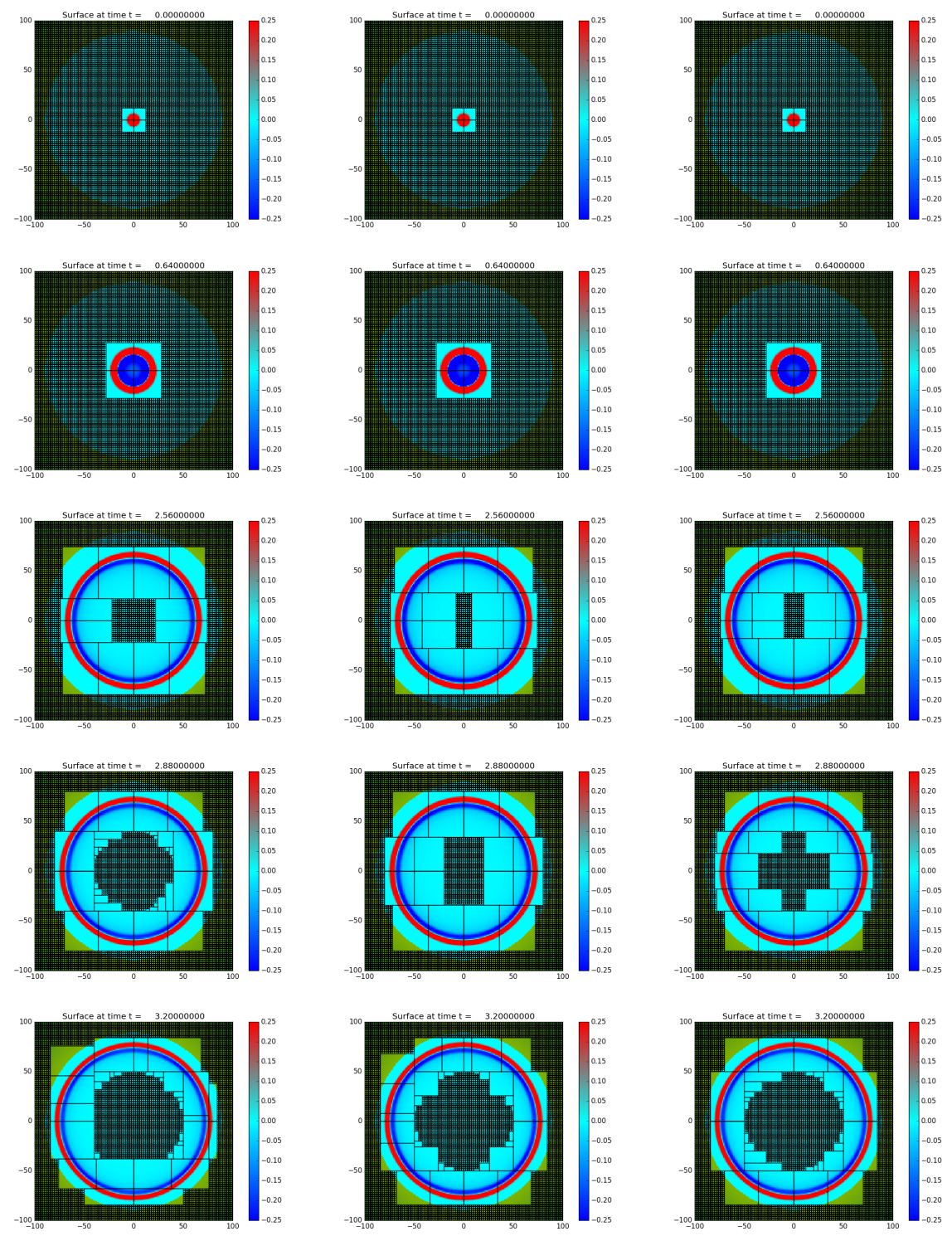

Figure 3: Radial bowl simulation showing generation of supermesh at the assimilation stage. Left - Ensemble member 1; Middle - Ensemble member 2; Right - Ensemble union generated 


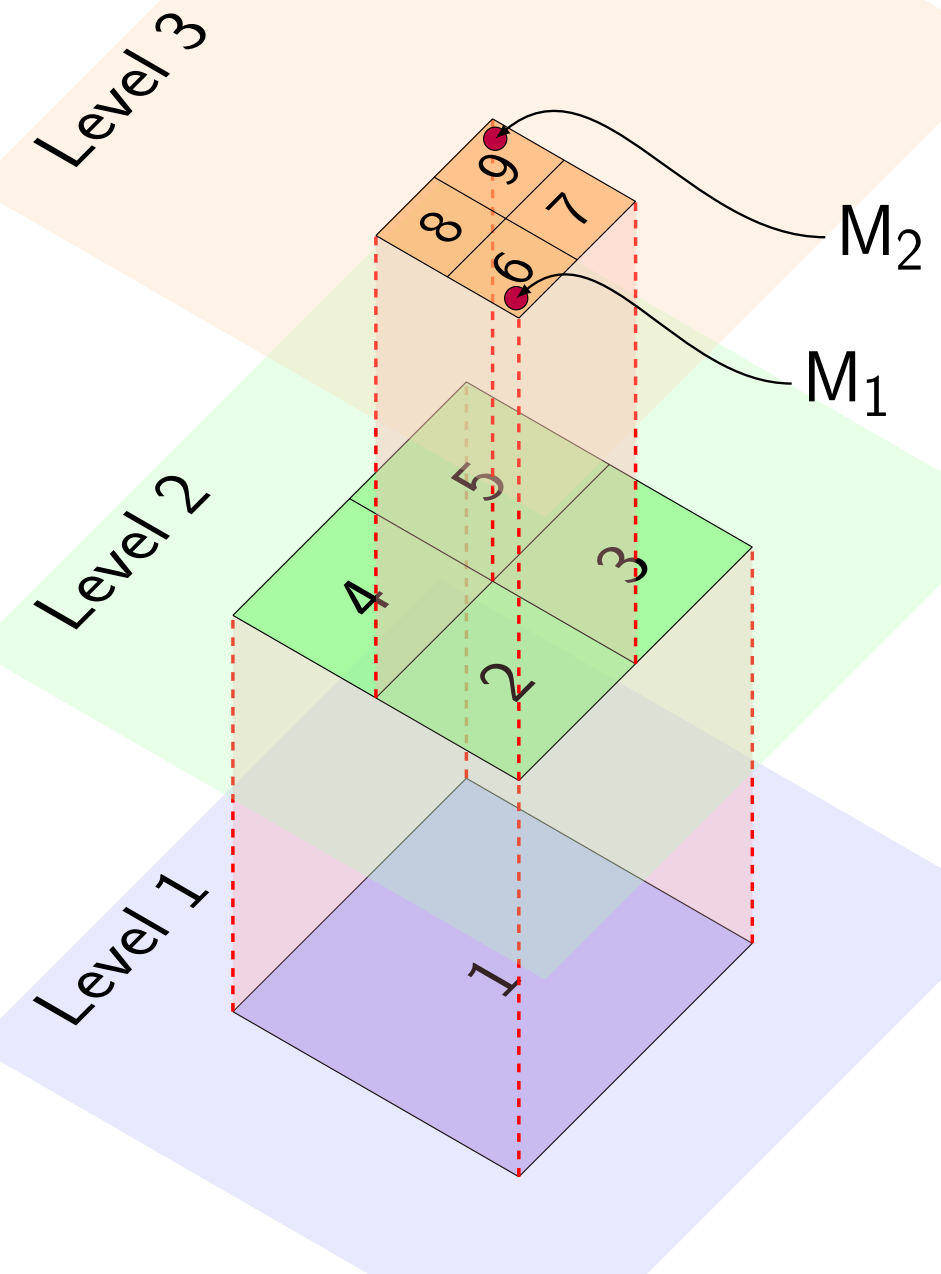

Figure 4: A simple 3 level mesh structure representing a state vector size of 9 elements along with 2 incoming observations $M_{1}$ and $M_{2}$. 


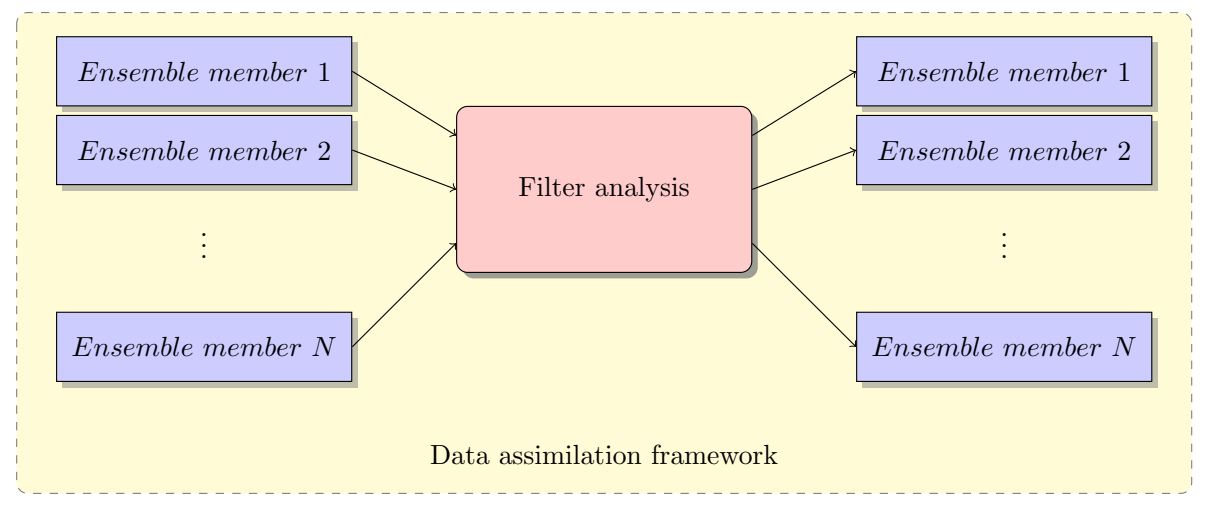

Figure 5: Parallelism in GEOCLAW-PDAF data assimilation framework 


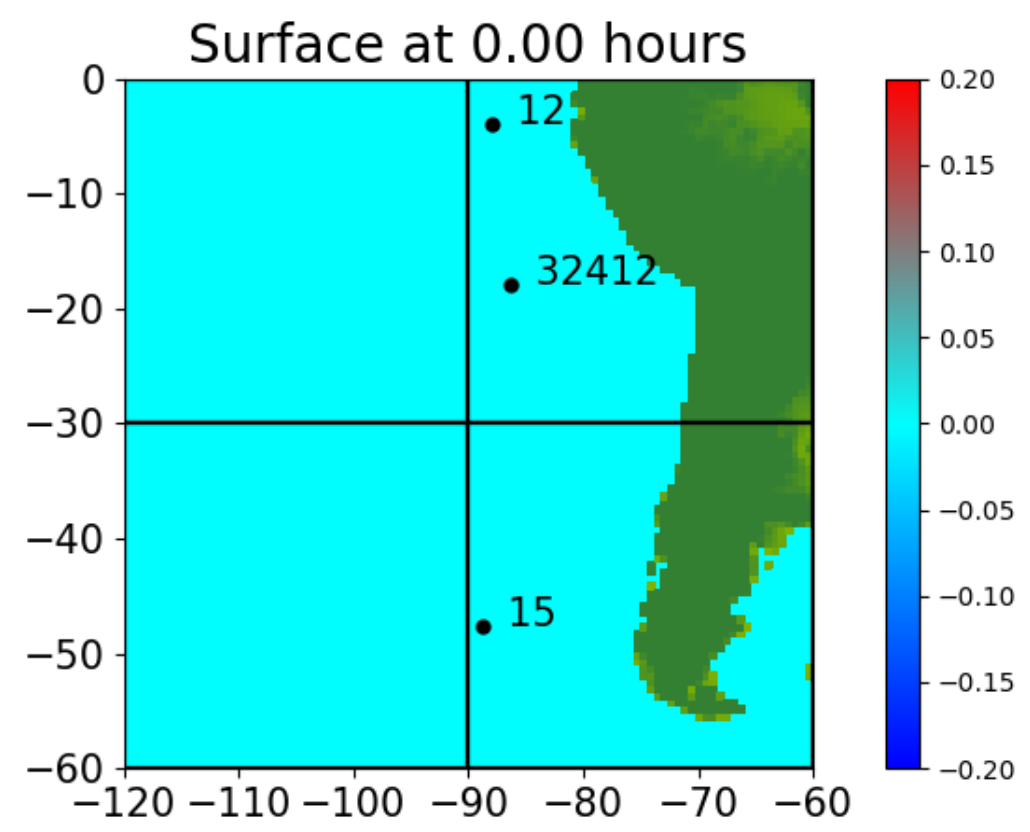

Figure 6: Computational domain for Chile February 27, 2010 tsunami simulation 


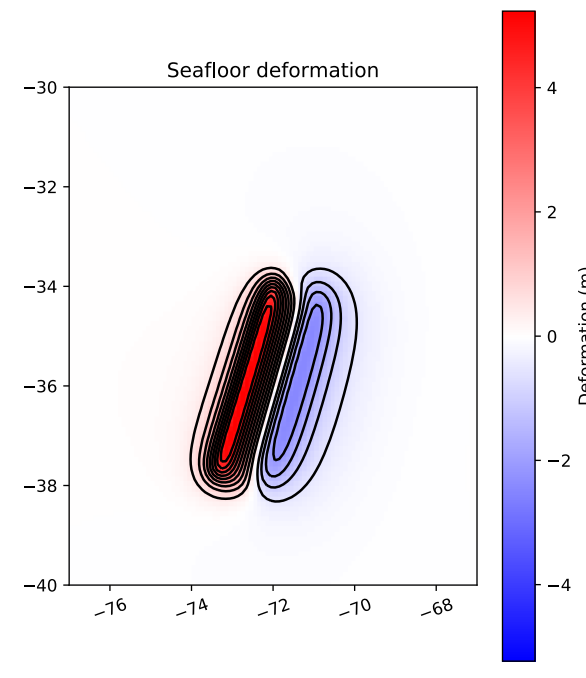

(a) "True" fault parameters

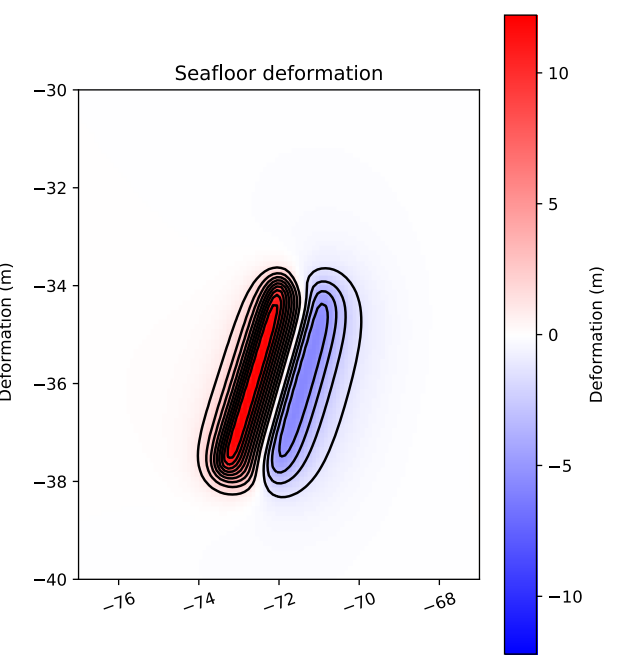

(b) Biased fault parameters

Figure 7: Bathymetry changes from the biased fault parameters in Table 2 

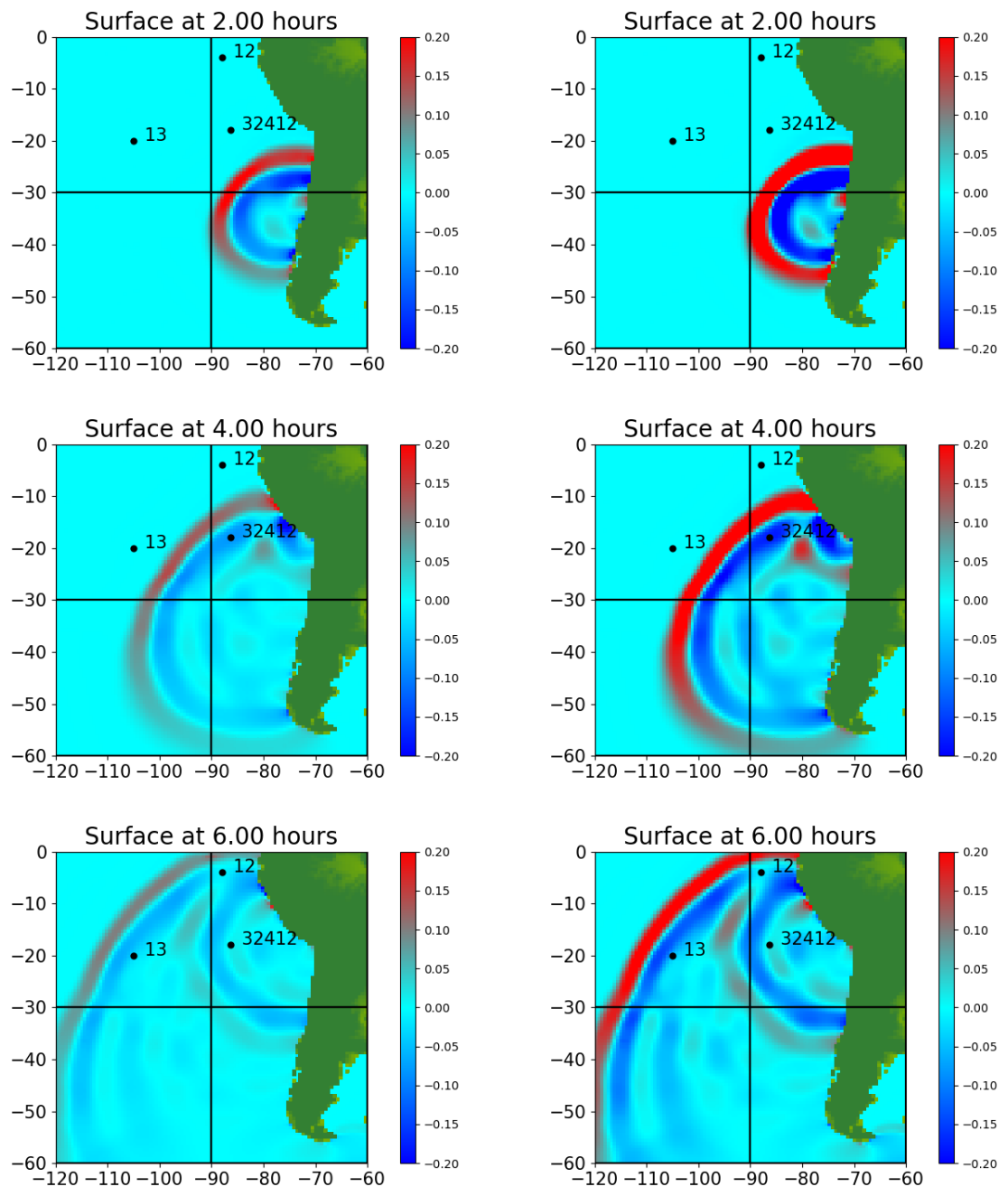

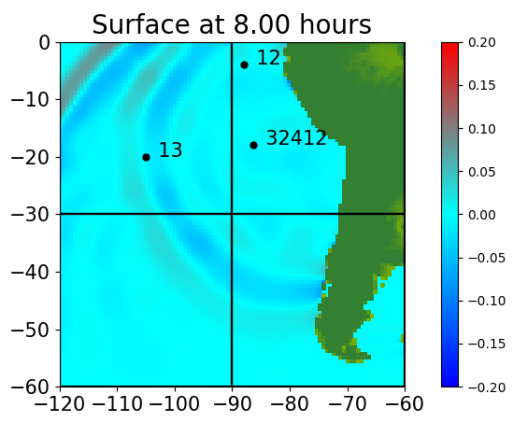

(a) "True" fault parameters

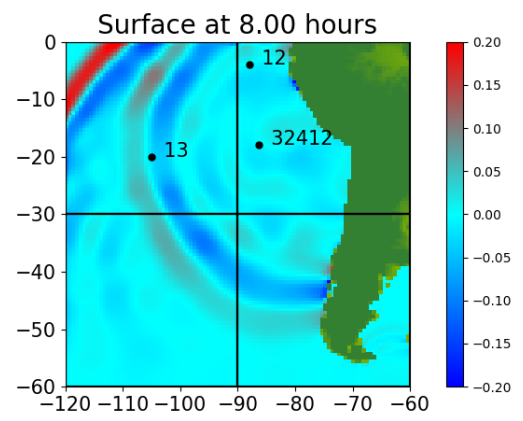

(b) Biased fault parameters

Figure 8: Snapshots of water surface elevation of the tsunami caused by bathymetry changes due to the fault parameters in Table 2 


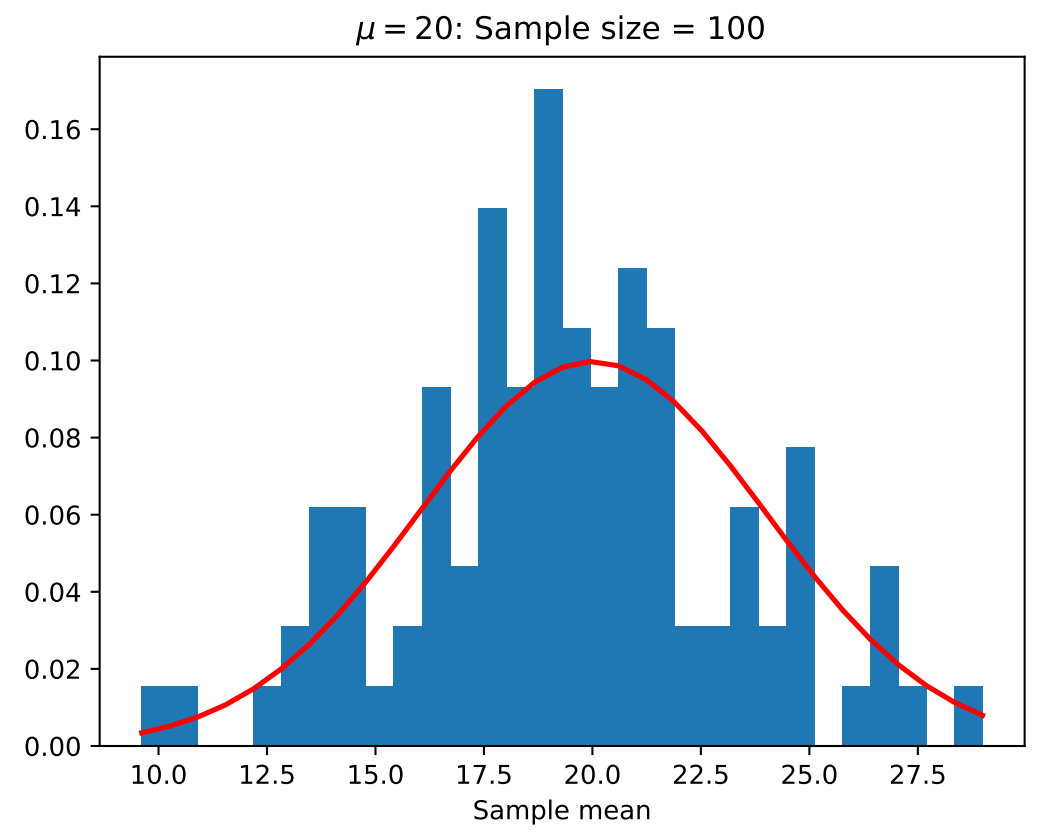

Figure 9: Sample distribution of slips for the initial ensemble generation algorithm 


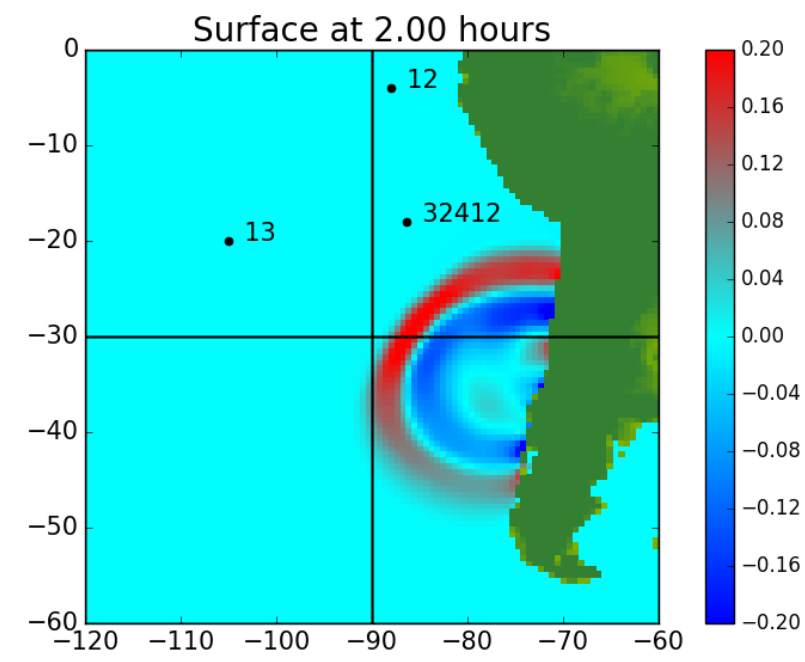

Figure 10: Water surface elevation at the checkpoint time of 2 hours. 

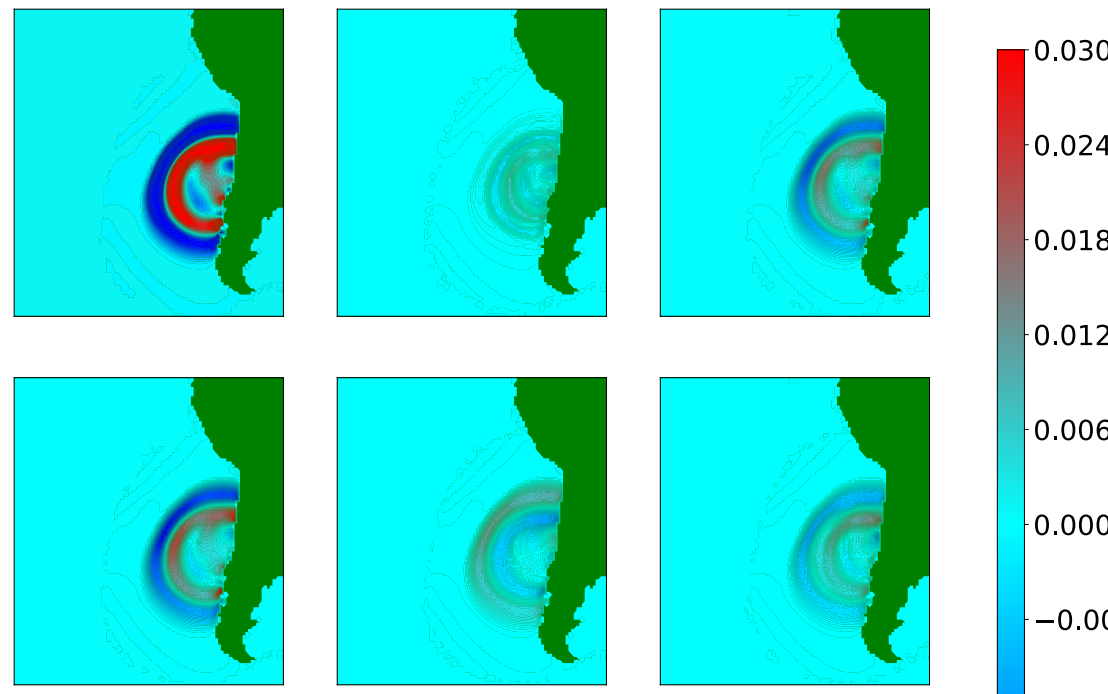

0.018
0.012

$\frac{t}{0}$

0.006

드

$0.000 \stackrel{\frac{0}{2}}{\frac{\pi}{2}}$

-0.006 岂
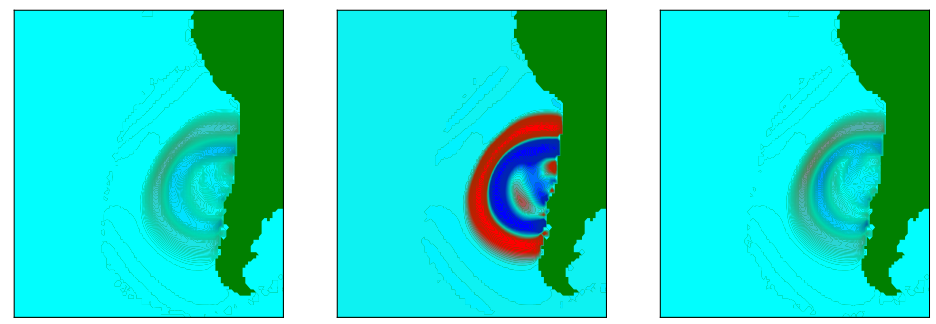

$-0.018 \stackrel{\frac{1}{ \pm}}{\frac{1}{\pi}}$

$-0.024$

$-0.030$

Figure 11: Perturbations in water surface elevation that will be added to the mean state at the checkpoint time $t_{c p}=2.0$ hours 


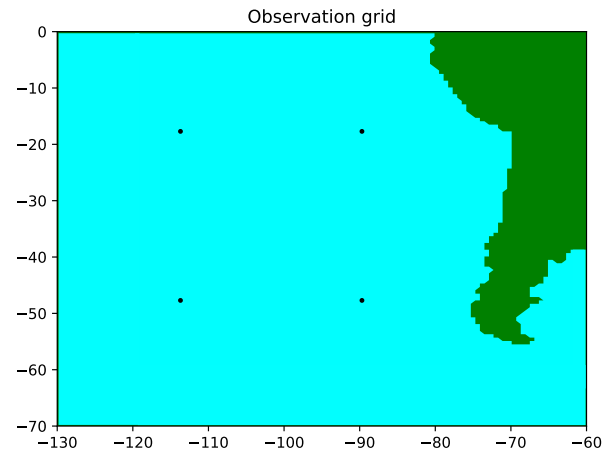

(a) 4 measurement sensors

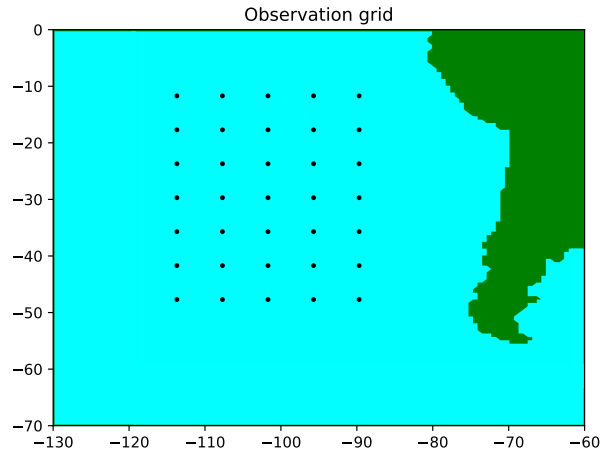

(c) 35 measurement sensors

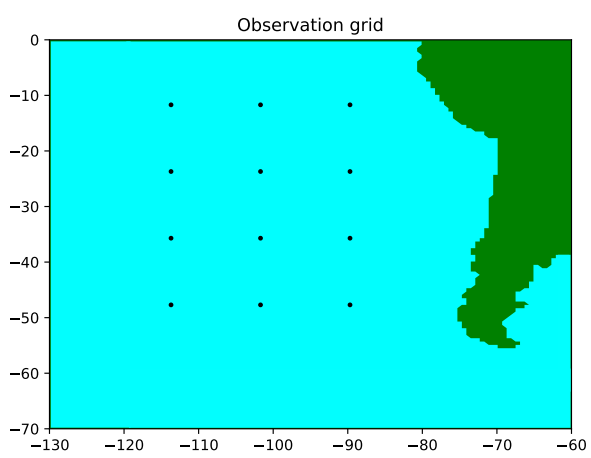

(b) 12 measurement sensors

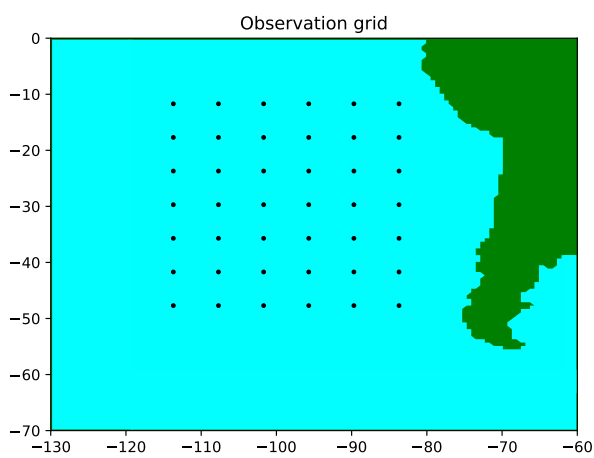

(d) 42 observation grid

Figure 12: Observation grid configurations for twin experiment 1 


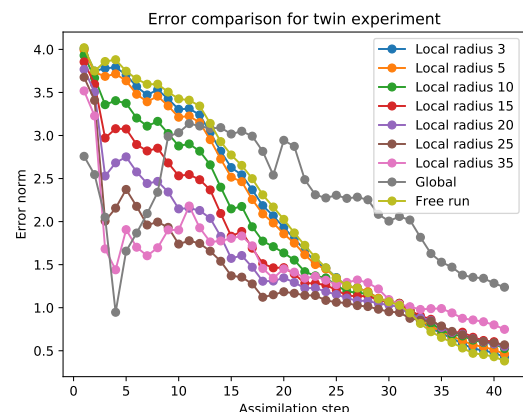

(a) Observation configuration of 4 sensors

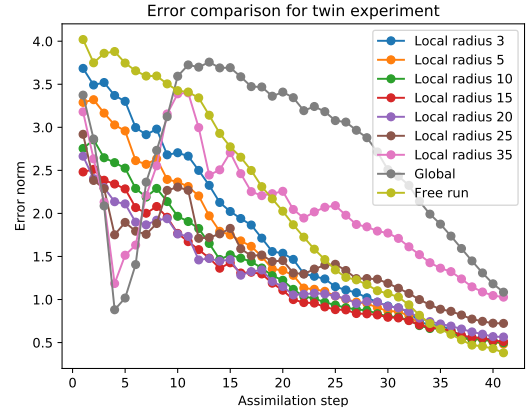

(c) Observation configuration of 35 sensors

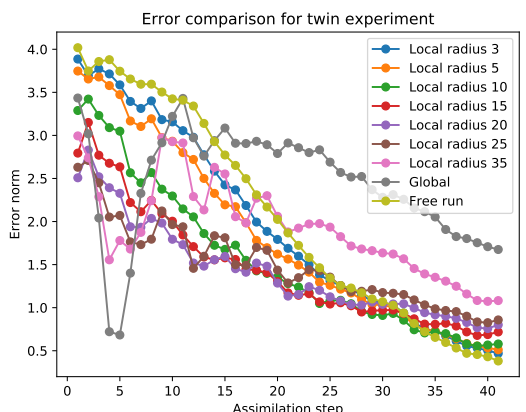

(b) Observation configuration of 12 sensors

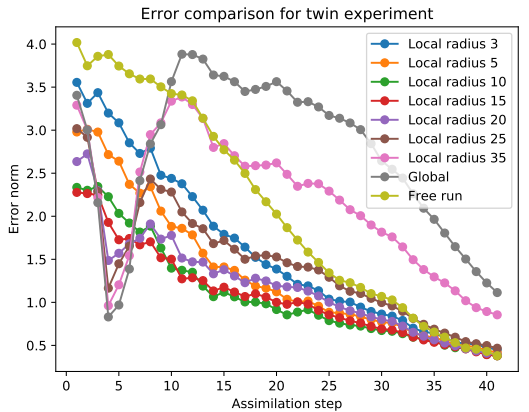

(d) Observation configuration of 42 sensors

Figure 13: Error comparison for twin experiment 1 with various observation configurations given in 12 

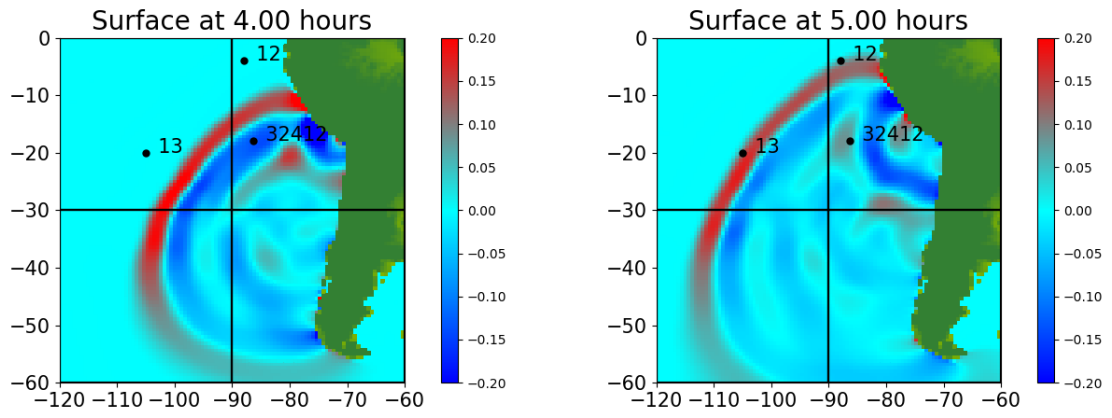

(a) 4 synthetic observations with the localization radius of $25^{\circ}$
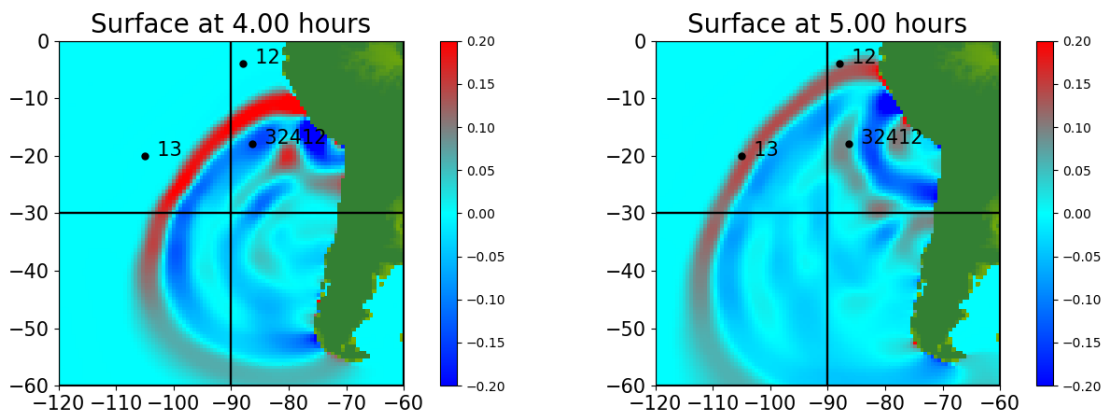

(b) 12 synthetic observations with the localization radius of $15^{\circ}$
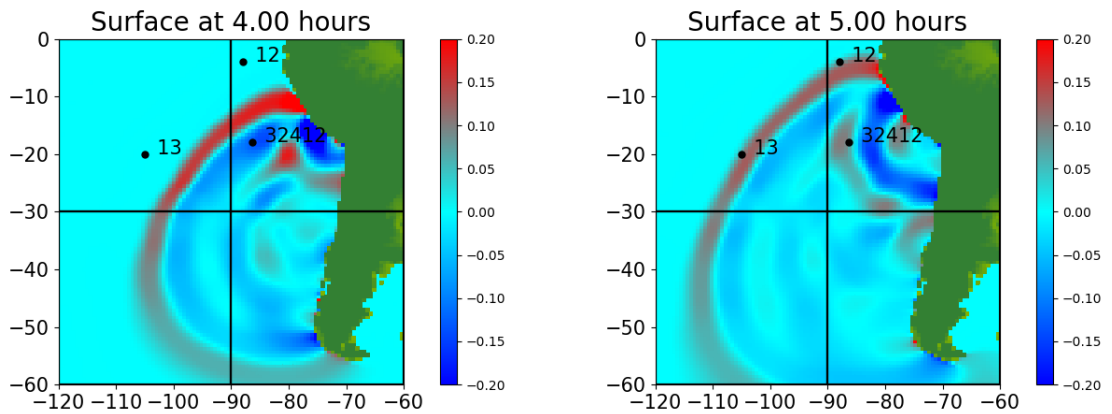

(c) 35 synthetic observationswith the localization radius of $15^{\circ}$
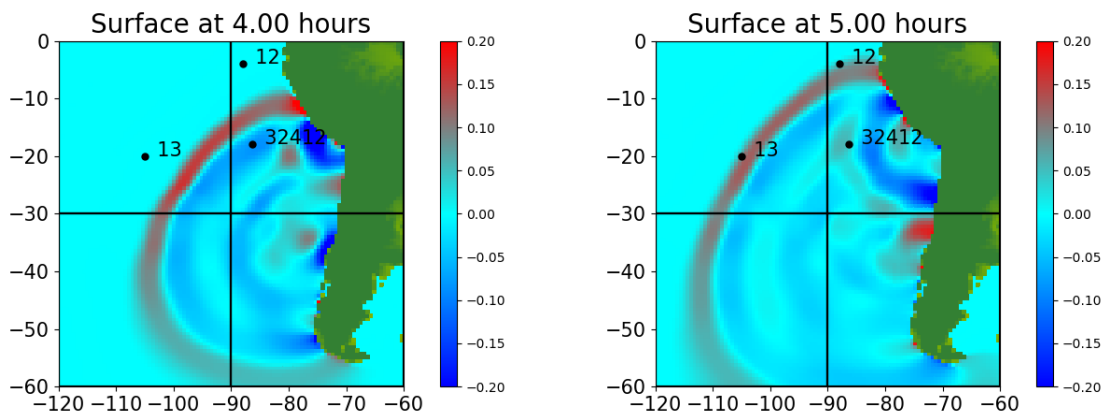

(d) 42 synthetic observations with the localization radius of $15^{\circ}$

Figure 14: Snapshots of mean water surface elevation in twin experiment 1 of Chile 2010 tsunami after synthetic observations are assimilated every 15 minutes. 


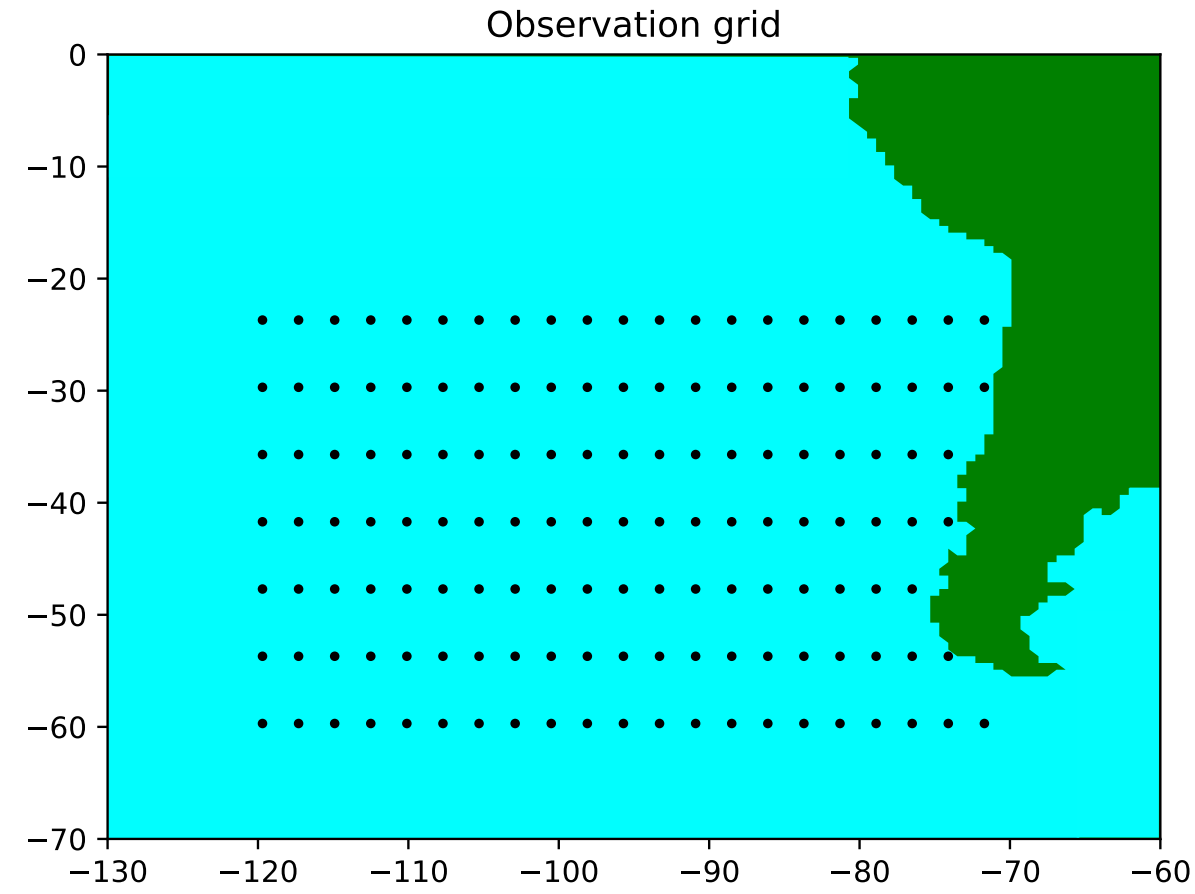

Figure 15: 142 observation points where synthetic measurements are generated for the twin experiment 2 of Chile tsunami simulation 


\section{Eigen value distribution}
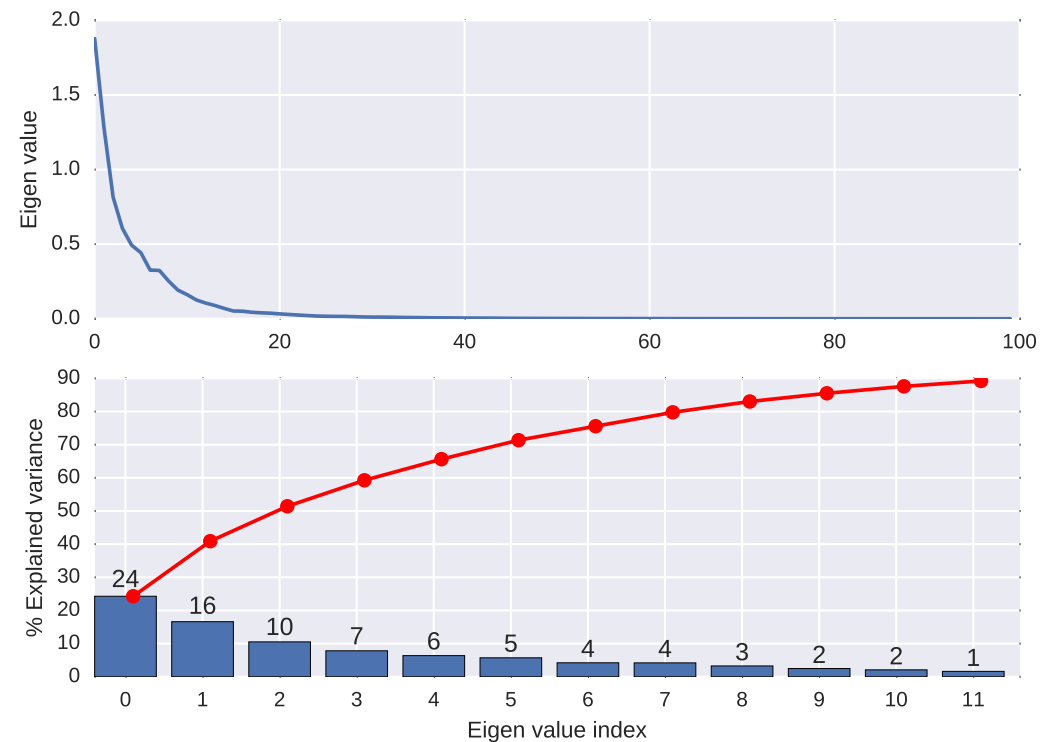

Figure 16: Eigen decomposition of 100 states of the possible tsunami outcomes at the checkpoint time of 2.0 hours for twin experiment 2 of February 272010 Chile tsunami data assimilation. 


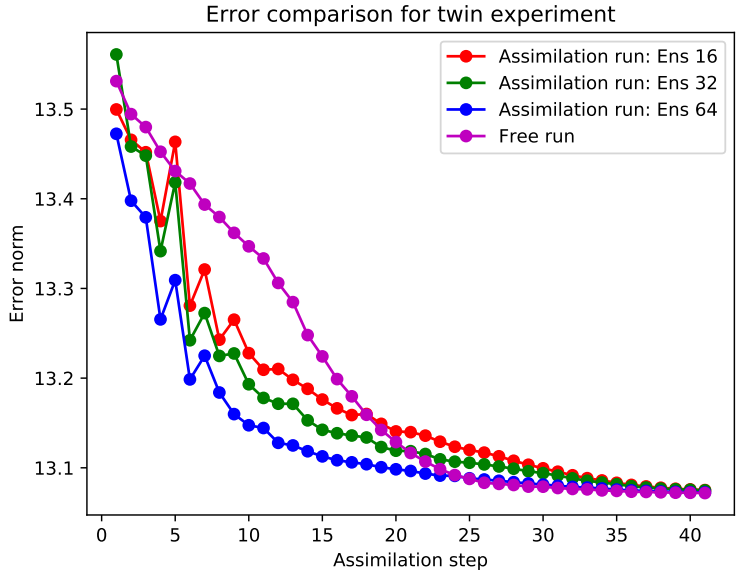

(a) Assimilation demonstrating use of 64 ensemble members to obtain errors between assimilated state and true state consistently less than errors between free run state and true state for every assimilation step

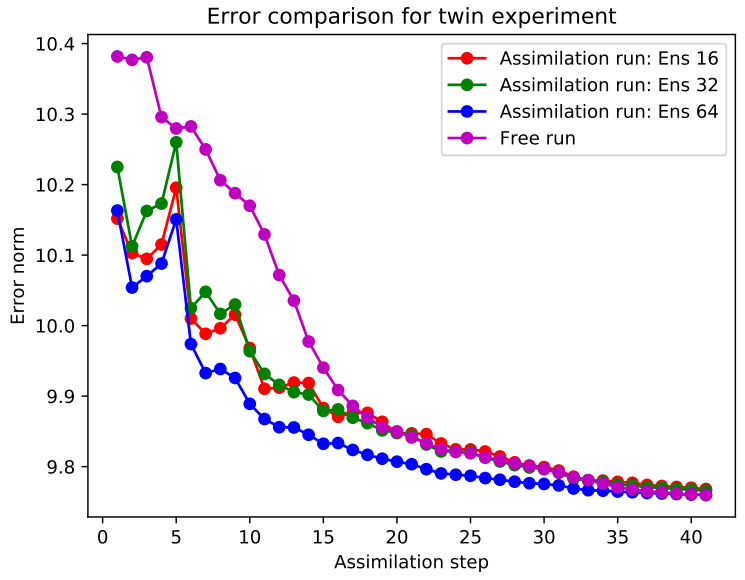

(b) Assimilation demonstrating use of 32 ensemble members to obtain errors between assimilated state and true state consistently less than errors between free run state and true state for every assimilation step

Figure 17: Effect of ensemble size on errors between true state and the global assimilated state with different sets of measurement values obtained from ensemble generation algorithm involving sampling of fault parameters as per Table 3 

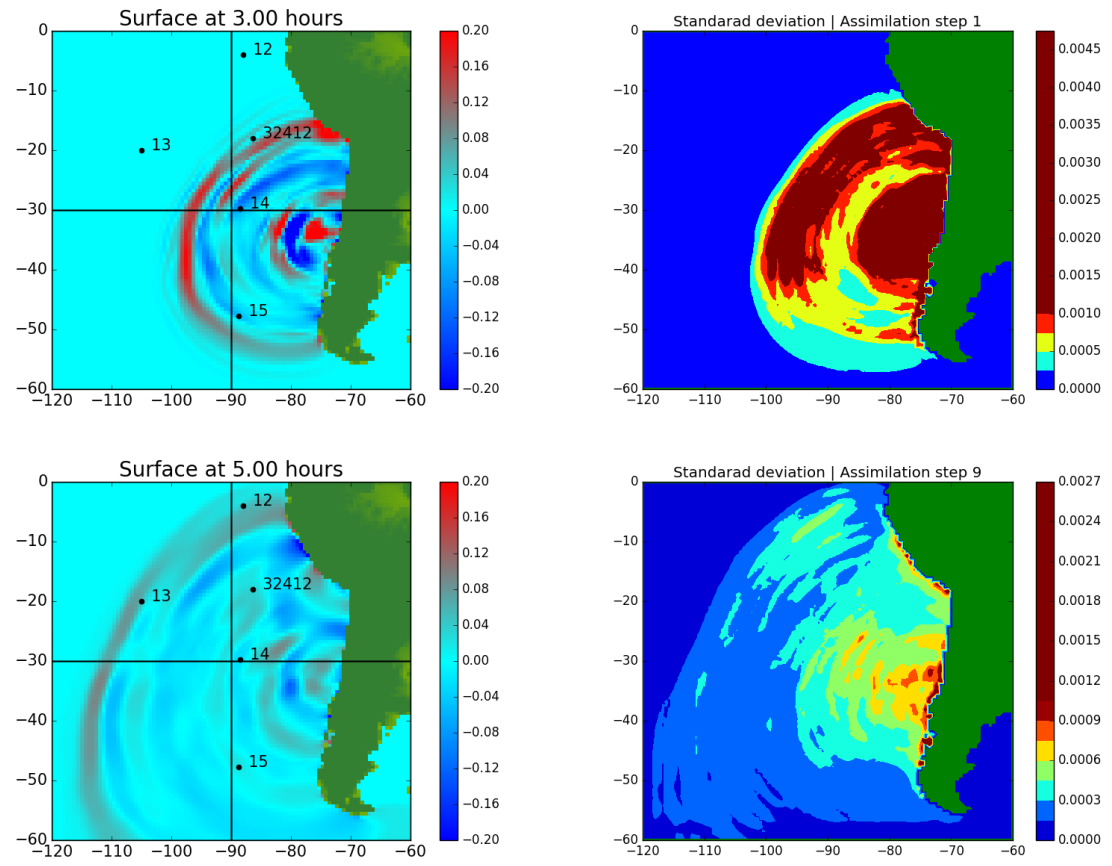

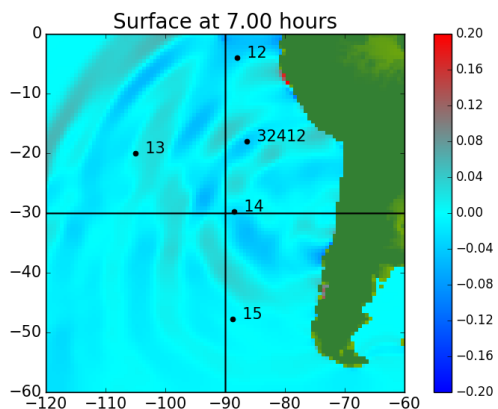

(a) Mean state

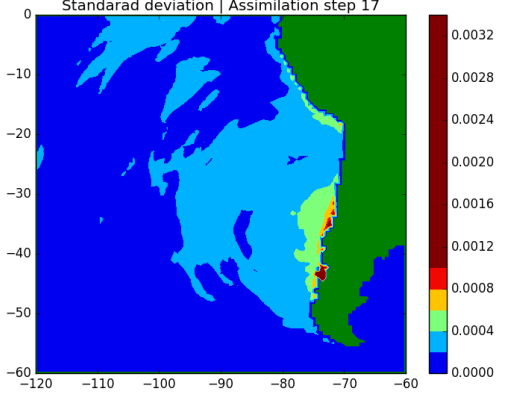

(b) RMSE

Figure 18: Snapshots of mean and RMSE of water surface elevation in twin experiment of Chile 2010 tsunami after 142 synthetic observations are assimilated every 15 minutes for twin experiment 2 

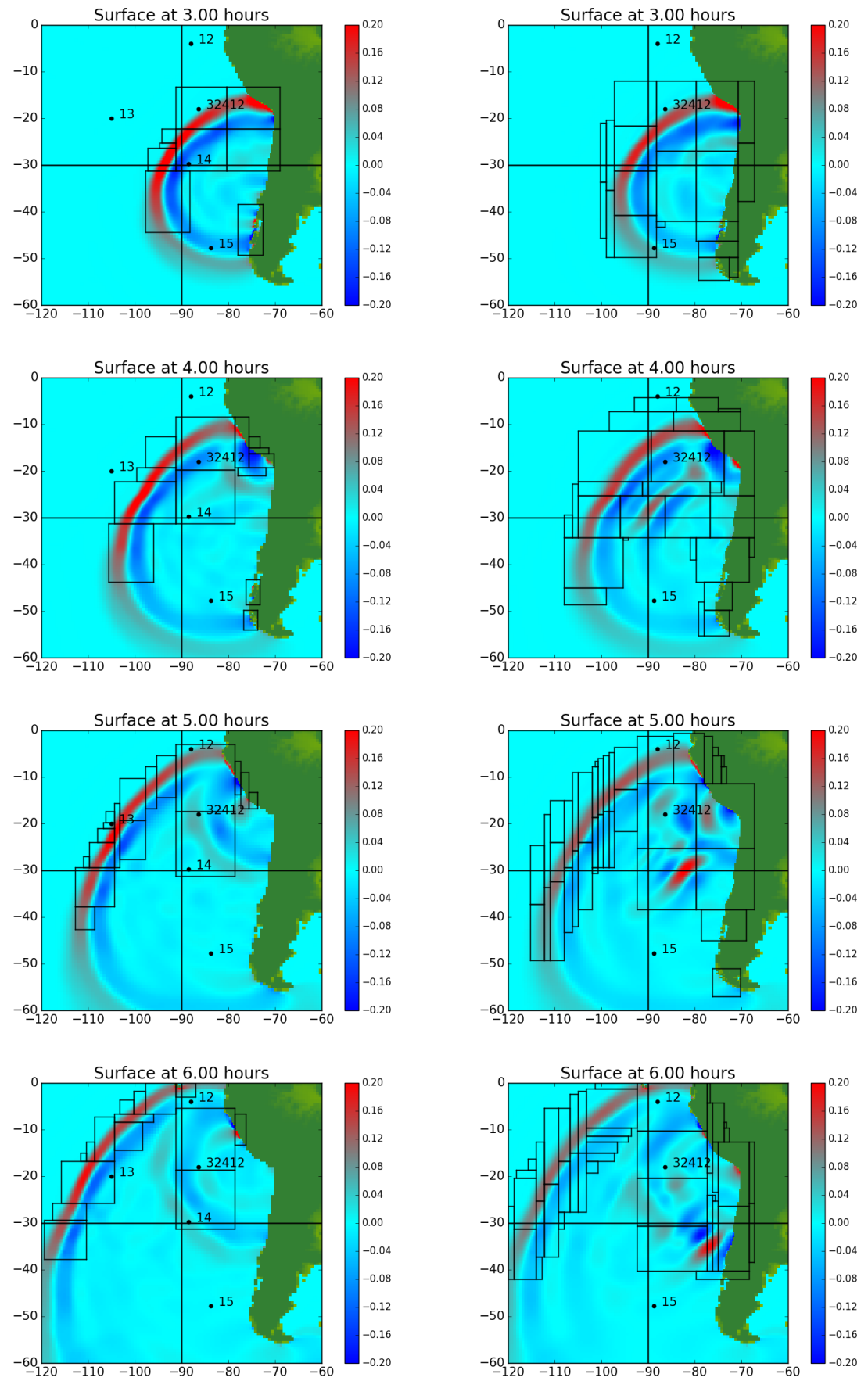

Figure 19: Water surface elevation comparison of (Right) mean assimilated state and (Left) state without assimilation after data from Gauge 32412 is assimilated every 15 minutes, with a maximum 2 level AMR 

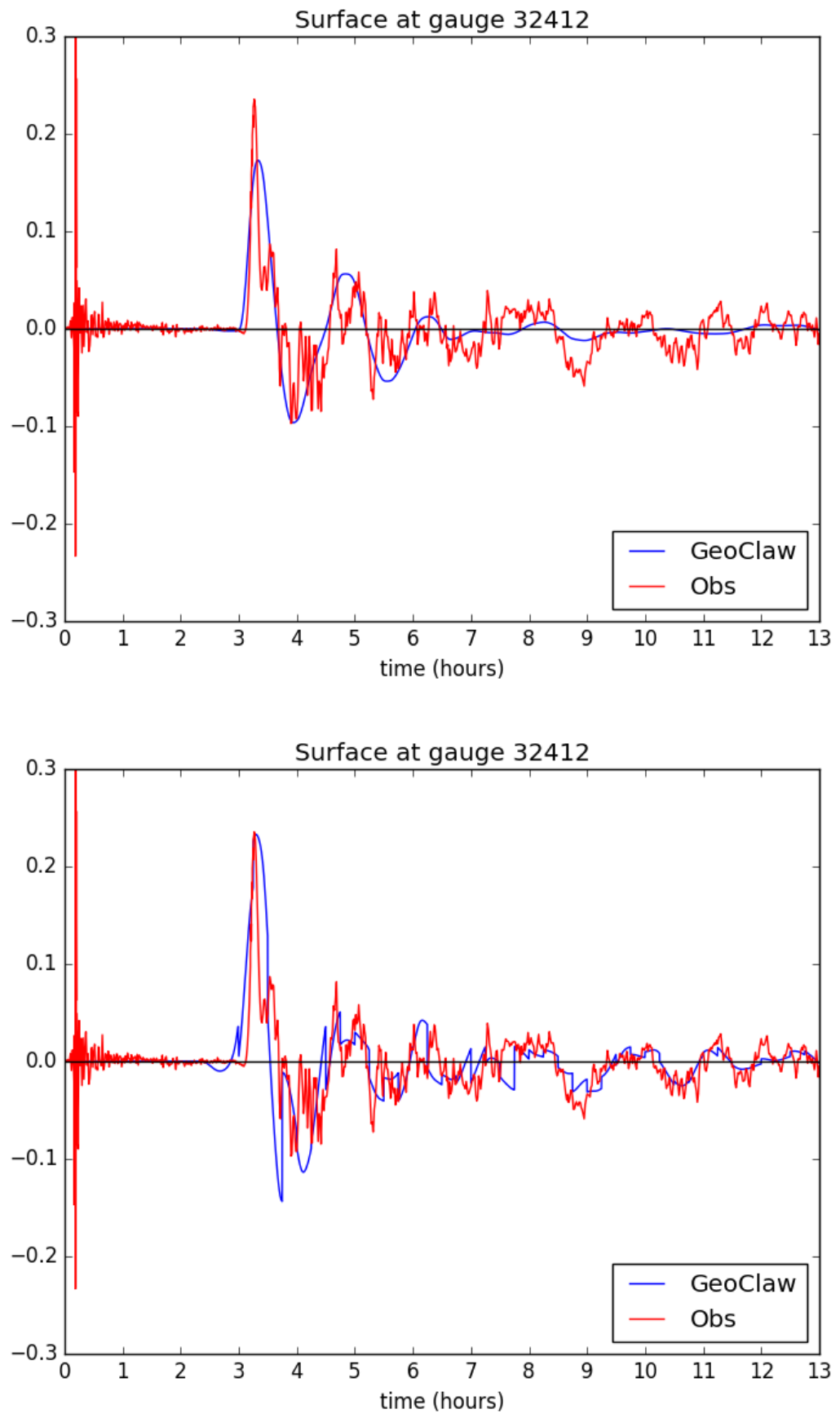

Figure 20: (Top) Gauge output for run without assimilation (Bottom) Gauge output for assimilation run of 16 ensemble members with localization radius of $20^{\circ}$ 